\title{
On Approximate Controllability of Second Order Fractional Impulsive Stochastic Differential System with Nonlocal, State-dependent Delay and Poisson Jumps
}

\author{
Krishnan Thiagu \\ Department of Mathematics, Kandaswami Kandar's College, Velur (Namakkal), Tamilnadu, India \\ Email address: \\ thiyaguelakk@gmail.com \\ To cite this article: \\ Krishnan Thiagu. On Approximate Controllability of Second Order Fractional Impulsive Stochastic Differential System with Nonlocal, \\ State-dependent Delay and Poisson Jumps. American Journal of Applied Mathematics. Vol. 9, No. 2, 2021, pp. $52-63$. \\ doi: 10.11648/j.ajam.20210902.13
}

Received: February 4, 2021; Accepted: March 30, 2021; Published: April 26, 2021

\begin{abstract}
The main scope of this paper is to focus the approximate controllability of second order $(q \in(1,2])$ fractional impulsive stochastic differential system with nonlocal, state-dependent delay and Poisson umps in Hilbert spaces. The existence of mild solutions is derived by using Schauder fixed point theorem. Sufficient conditions for the approximate controllability are established by under the assumptions that the corresponding linear system is approximately controllable and it is checked by using Lebesgue dominated convergence theorem. The main results are completly based on the results that the existence and approximate controllability of the fractional stochastic system of order $1<\mathrm{q} \leq 2$ and are derived by using stochastic analysis theory, fixed point technique, q-order cosine family $\left\{C_{a}(t)\right\}_{\unrhd \geq 0}$, new set of novel sufficient conditions and methods adopted directly from deterministic fractional equations for the second order nonlinear impulsive fractional nonlocal stochastic differential systems with state-dependent delay and Poisson jumps in Hildert space H. Finally an example is added to illustrate the main results.
\end{abstract}

Keywords: Approximate Controllability, Fixed-Point Theorem, Fractional Stochastic Differential System, Hilbert Space, Poisson Jumps

\section{Introduction}

The concept of semigroups of bounded linear operators is taken as an important concept to dealing with differential and integro-differential equations in Banach spaces [8, 14, 15, 17, 36]. For more points of interest on this concept, we refer to Pazy [36]. On the other hand, in numerous mathematical models of real world or man made phenomena, we are led to dynamical systems which involve some inherent randomness. These systems are called stochastic systems. Stochastic differential equations [34] have attracted much attention and have played an important role in many ways such as option pricing, forecast of the growth of population, etc [16, 18, 29, 34]. In the last few decades, fractional differential systems (we refer to the monographs $[28,31,35,41]$ and references cited therein) have focused considerable importance in electrochemistry, physics, porous media, control theory, engineering etc., $[4,5,6,10,11,44]$ due to the descriptions of memory and hereditary properties of various materials and processes. Notion of controllability is of great importance in mathematical control theory due to a number of important properties of control systems in engineering. Astrom [1] discussed about introduction to stochastic control theory. In the infinite dimensional systems, two basic concepts of controllability are exact and approximate controllability. Exact controllability enables to steer the system to arbitrary final state while approximate controllability is weaker concept of controllability and it is possible to steer the system to an arbitrary small neighborhood of the final state (see, for example, [2, 9, 12, 46, 50]). Impulsive effects [47] exist widely in many evolution process because, the impulsive effects may bring an abrupt change at a certain moments of time involving such fields as economics, mechanics, electronics, telecommunications, medicine and biology, etc. Kexue et al. [27] studied controllability of nonlocal fractional differential systems of order $\alpha \in(1,2]$ in Banach spaces. Delay fractional differential equations are similar to fractional differential equations, but their evolution involves past value of the state variable. Muthukumar and Thiagu [32] proved the existence of solutions and approximate controllability of fractional nonlocal stochastic differential equations of order 
$q \in(1,2]$ with infinite delay and Poisson jumps in Hilbert spaces by using fixed point theory and natural assumption that the corresponding linear system is approximately controllable. Hence problem of existence of approximate controllability for nonlinear fractional impulsive stochastic differential equations with nonlocal conditions and infinite delay has been studied by several authors were received significant attention in modern days (see [10, 12, 33, 40, 49] and references therein). Rajivganthi et al. [38] studied existence of solutions and approximate controllability of impulsive fractional stochastic differential systems with infinite delay and poisson jumps.

Many authors (see [21, 22, 23, 30, 39]) established the existence and approximate controllability of different types of functional differential equations with state-dependent delay. Fractional differential equations with state-dependent delay appear frequently in applications as models of equations and for this reason the study of this type of equations has been receiving great attention in recent years (see [3, 7, 43, 45] and references therein). Many authors (see [13, 19, 26, 48, 51] and references therein) studied the existence and approximate controllability as well as stability of different types of fractional stochastic differential equations with state-dependent delay in Hilbert spaces under different suitable aspects. Selvarasu et al. [41] established approximate controllability of impulsive fractional stochastic integro-differential systems with state-dependent delay and poisson Jumps of order $1<q<2$. Moreover approximate controllability results for fractional impulsive stochastic differential system of order $q \in(1,2]$ with nonlocal, state-dependent delay and Poisson jumps in Hilbert spaces has not yet been derived in the literature. The main purpose of this paper is to obtain the sufficient conditions of approximate controllability results for fractional impulsive stochastic differential system of order $q \in(1,2]$ with nonlocal, state-dependent delay and Poisson jumps in Hilbert spaces of the form

$$
\begin{gathered}
{ }^{c} D_{t}^{q} x(t)=A x(t)+B u(t)+f\left(t, x_{\rho\left(t, x_{t}\right)}\right) \\
+\int_{-\infty}^{t} g\left(t, x_{\rho\left(t, x_{t}\right)}\right) d \omega(t)+\int_{Z} \mathfrak{h}\left(t, x_{\rho\left(t, x_{t}\right)}, \eta\right) \widetilde{N}(d t, d \eta) \\
t \in J:=[0, b] \backslash\left\{t_{1}, \ldots, t_{n}\right\}, 0=t_{0}<t_{1}<\cdots<t_{n}<t_{n+1}= \\
b, \\
x_{0}(t)=\phi(t)+\mathbf{m}\left(x_{t_{1}}, \ldots, x_{t_{m}}\right)(t), \\
(m>n), m, n \in \mathbb{N}, \\
x^{\prime}(0)=\xi \\
\Delta x\left(t_{k}\right)=I_{k}\left(x_{t_{k}}\right), \\
\Delta x^{\prime}\left(t_{k}\right)=\bar{I}_{k}\left(x_{t_{k}}\right), k=1,2, \ldots, n:=\overline{1, n} .
\end{gathered}
$$

Here, the state variable $x(\cdot)$ takes values in a real separable Hilbert space $H$ with the inner product $\langle\cdot, \cdot\rangle$ and norm $\|\cdot\|_{H}$. The fractional derivative ${ }^{c} D_{t}^{q}, 1<q \leq 2$ is understood in the Caputo sense. Let $0=t_{0}<t_{1}<\cdots<t_{n}<t_{n+1}=b$ be the given time points. The control function $u(\cdot)$ is given in $\mathcal{L}_{2}(J, U)$ of admissible control functions with $U$ as a Hilbert space. $B$ is a bounded linear operator from $U$ into $H$. Also $A: D(A) \subset H \rightarrow H$ is the infinitesimal generator of a strongly continuous cosine family $C(t)$ on $H$. Let $K$ be another separable Hilbert space. Let $\{\omega(t)\}_{t \geq 0}$ be a given $K$-valued Wiener process with a finite trace nuclear covariance operator $Q \geq 0$. Let $\tilde{q}=\left\{\tilde{q}(t): t \in D_{\tilde{q}}\right\}$ be a stationary $\mathfrak{F}_{t}$ Poisson point process with characteristic measure $\lambda$. Let $N(d t, d \eta)$ be the Poisson counting measure associated with $\tilde{q}$. Then $N(t, Z)=\sum_{s \in D \widetilde{q}, s \leq t} I_{Z}(\tilde{q}(s))$ with measurable set $Z \in \widetilde{B}(K-\{0\})$, which denotes the Borel $\sigma$-field of $. K-\{0\}$ Let $\widetilde{N}(d t, d \eta)=N(d t, d \eta)-d t \lambda(d \eta)$ be the compensated Poisson measure that is independent of $\omega(t)$. Let $P_{2}([0, b] \times$ $Z ; H)$ be the space all mapping $\chi:[0, b] \times Z \rightarrow H$ for which $\int_{0}^{b} \int_{Z} \mathbb{E}\|\chi(t, \eta)\|_{H}^{2} d t \lambda(d \eta)<\infty$. We can define the H-valued stochastic integral $\int_{0}^{b} \int_{Z} \chi(t, \eta) \widetilde{N}(d t, d \eta)$, which is a centered square integrable martingale. We can also employing the same notation $\|\cdot\|$ for the norm of $\mathcal{L}(K, H)$, which denotes the space of all bounded operators from $K$ into $H$. Simply $\mathcal{L}(H)$ if $K=H$. The histories $x_{t}$ represents the function defined by $x_{t}:(-\infty, 0] \rightarrow H, x_{t}(\theta)=x(t+\theta)$, for $t \geq 0$ belong to some phase space $\mathcal{B}$ described axiomatically and $\rho: J \times \mathcal{B} \rightarrow(-\infty, b]$ is a continuous function. Further $f: J \times \mathcal{B} \rightarrow H, \quad g: J \times \mathcal{B} \rightarrow \mathcal{L}_{Q}(K, H)$ and $\mathfrak{h}: J \times \mathcal{B} \times Z \rightarrow$ $H$ are nonlinear functions. Here $\mathcal{L}_{Q}(K, H)$ denotes the space of all $Q$-Hilbert Schmidt operators from $K$ into $H$. Let $P C\left(J, \mathcal{L}_{2}(\Omega, \mathfrak{F}, \mathbb{P} ; H)\right)=\{x(t)$ is continuous everywhere except for some $t_{k}$ at which $x\left(t_{k}^{-}\right)$and $x\left(t_{k}^{+}\right)$exist and $\left.x\left(t_{k}^{-}\right)=x\left(t_{k}\right), \quad k=1,2, \ldots, m\right\}$ be the Banach space of piece-wise continuous function from $J$ into $\mathcal{L}_{2}(\Omega, \mathfrak{F}, \mathbb{P} ; H)$ with the norm $\|x\|_{P C}=\sup _{t \in J}|x(t)|<\infty . P C\left(J, \mathcal{L}_{2}\right)$ is the closed subspace of $P C\left(J, \mathcal{L}_{2}(\Omega, \mathfrak{F}, \mathbb{P} ; H)\right)$ consisting of measurable and $\mathfrak{F}_{t}$-adapted $H$-valued processes $x(\cdot) \in$ $P C\left(J, \mathcal{L}_{2}(\Omega, \mathfrak{F}, \mathbb{P} ; H)\right)$ endowed with the norm $\|x\|^{2}=$ $\sup \left\{\mathbb{E}\|x(t)\|^{2}, t \in J\right\}$. Moreover the function $\mathbf{m}: H^{m} \rightarrow$ $H$, where $0=t_{0}<t_{1}<t_{2}<\ldots<t_{m} \leq T, \quad m \in \mathbb{N}$ is a continuous function. For example (refer [25])

$$
\mathbf{m}\left(x_{t_{1}}, \ldots, x_{t_{m}}\right)(t)=\sum_{i=0}^{m} C_{i} z\left(t_{i}+t\right), x \in \mathcal{B}, t \geq 0,
$$

where $C_{i}(i=1,2, \ldots, m)$ are constants. $I_{k}$ and $\bar{I}_{k}: H \rightarrow$ $H$ are appropriate functions. The jump in the state $x$ at time $t_{k}$ is defined by $\Delta x=x\left(t_{k}^{+}\right)-x\left(t_{k}^{-}\right)$. The initial data $\phi=\{\phi(t): t \in(-\infty, 0]\}$ is an $\mathfrak{F}_{0}$-measurable $\mathcal{B}$-valued stochastic process independent of Brownian motion $\{\omega(t)\}$ and Poisson point process $\tilde{q}(\cdot)$ with finite second moment. Further $\xi(t)$ is an $\mathfrak{\mho}_{t}$-measurable $H$-valued random variable independent of $\omega(t)$ and Poisson point process $\tilde{q}$ with finite second moment.

\section{Preliminaries}

Let $(\Omega, \mathfrak{F}, \mathbb{P})$ be a complete probability space furnished with complete family of right continuous increasing sub $\sigma$-algebras $\left\{\mathfrak{F}_{t}: t \in J\right\}$ satisfying. $\mathfrak{F}_{t} \subset \mathfrak{F}$. An $H$-valued random variable is an $\mathfrak{F}$-measurable function $x(t): \Omega \rightarrow$ 
$H$ and a collection of random variables $S=\{x(t, w): \Omega \rightarrow$ $\left.\left.H\right|_{t \in J}\right\}$ is called a stochastic process. Usually, we suppress the dependence on $w \in \Omega$ and write $x(t)$ instead of $x(t, w)$ and $x(t): J \rightarrow H$ in the place of $S$. Let $\beta_{n}(t)(n=1,2, \ldots)$ be a sequence of real valued one-dimensional standard Brownian motions mutually independent over $(\Omega, \mathfrak{F}, \mathbb{P})$. Set $\omega(t)=\sum_{n=1}^{\infty} \sqrt{\lambda_{n}} \beta_{n}(t) e_{n}, t \geq 0$, where $\lambda_{n} \geq 0 \quad(n=$ $1,2, \ldots)$ are non-negative real numbers and $\left\{e_{n}\right\} \quad(n=$ $1,2, \ldots)$ is complete orthonormal basis in $K$. Let $Q \in$ $\mathcal{L}(K, K)$ be an operator defined by $Q e_{n}=\lambda_{n} e_{n}$ with finite $\operatorname{Tr}(Q)=\sum_{n=1}^{\infty} \lambda_{n}<\infty, \operatorname{Tr}(Q)$ denotes the trace of the operator $Q$. Then the above $K$-valued stochastic process $\omega(t)$ is called a $Q$-Wiener process. Let us assume that $\mathfrak{F}_{t}=\sigma(\omega(s): 0 \leq s \leq t)$ is the $\sigma$-algebra generated by $\omega$ and $. \mathfrak{F}_{t}=\mathfrak{F}$. Let $\varphi \in \mathcal{L}(K, H)$ and define $\|\varphi\|_{Q}^{2}-$ $\operatorname{Tr}\left(\varphi Q \varphi^{\star}\right)=\sum_{n=1}^{\infty}\left\|\sqrt{\lambda_{n}} \varphi e_{n}\right\|^{2}$. If $\|\varphi\|_{Q}<\infty$, then $\varphi$ is called a $Q$-Hilbert Schmidt operator. Let $\mathcal{L}_{Q}(K, H)$ denotes the space of all $Q$ Hilbert Schmidt operators $\varphi \in \mathcal{L}(K, H)$. The completion $\mathcal{L}_{Q}(K, H)$ of $\mathcal{L}(K, H)$ with respect to the topology induced by a norm $\|\cdot\|_{Q}$, where $\|\varphi\|_{Q}^{2}=\langle\varphi, \varphi\rangle$ is a Hilbert space with the above norm topology. Let $\mathcal{C}$ be the closed subspace of all continuously differentiable process that belong to the $\operatorname{space} \mathcal{C}\left(J, \mathcal{L}_{2}(\Omega ; H)\right)$ consisting of $\mathfrak{F}_{t}$-adapted measurable process such that $\mathfrak{F}_{0}$-adapted processes $\phi$, $\xi \in \mathcal{L}_{2}(\Omega, \mathcal{B})$.

In this work we will employ an axiomatic definition of the phase space $\mathcal{B}$ introduced by Hale and Kato [20]. The axioms of the space $\mathcal{B}$ are established for $\mathcal{F}_{?}$ - measurable functions from $J_{0}$ into $H$, where $J_{0}:=(-\infty, 0]$, endowed with a seminorm $\|\cdot\|_{H}$. We will assume that $\mathcal{B}$ satisfies the following axioms:

(a) If $x:(-\infty, b) \rightarrow H, b>0$ is continuous on $[0, b)$ and $x_{0} \in \mathcal{B}$, then for each $t \in[0, b)$ the following conditions hold,

(I) $x_{0} \in \mathcal{B}$,

(ii), $\|x(t)\| \leq K_{1}\left\|x_{t}\right\|_{\mathcal{B}}$,

(iii) $\left\|x_{t}\right\|_{\mathcal{B}} \leq K_{2}(t)\left\|x_{0}\right\|_{\mathcal{B}}$

$+K_{3}(t) \sup \{\|x(s)\| ; 0 \leq s \leq b\}$, where $K_{1}>0$ is a constant, $K_{2}:[0, \infty) \rightarrow[0, \infty)$ is a locally bounded function, $K_{3}:[0, \infty) \rightarrow[0, \infty)$ is a continuous function. Moreover $K_{1}, K_{2}(\cdot), K_{3}(\cdot)$ are independent of $x(\cdot)$,

(b) For the function $x(\cdot)$ in (a), $x_{t}$ is a $\mathcal{B}$-valued continuous functions on, $[0, b)$,

(c) The space $\mathcal{B}$ is complete.

Examples of phase space satisfying the above axioms can be found in Hale et al. And Hernandez et al. [21,24]. The $\mathcal{B}$-valued stochastic process $x_{t}: \Omega \rightarrow \mathcal{B}, t \geq 0$, is defined by setting

$$
x_{t}=\{x(t+s)(w): s \in(-\infty, 0]\} .
$$

The collection of all strongly measurable, square integrable $H$ valued random variables, denoted by $\mathcal{L}_{2}(\Omega, \mathfrak{F}, \mathbb{P} ; H) \equiv$ $\mathcal{L}_{2}(\Omega ; H)$,is a Banach space equipped with norm $\|x(\cdot)\|_{\mathcal{L}_{2}}=$ $\left(\mathbb{E}\|x(\cdot ; w)\|_{H}^{2}\right)^{\frac{1}{2}}$, where the expectation, $\mathbb{E}$ is defined by $\mathbb{E}(h)=\int_{\Omega} h(w) d \mathbb{P}$. Let $J_{1}=(-\infty, b]$ and $C\left(J_{1}, \mathcal{L}_{2}(\Omega ; H)\right)$ be the Banach space of all continuous maps from $J_{1}$ into $\mathcal{L}_{2}(\Omega ; H)$ satisfying the condition.sup $\mathbb{t}_{t \in J_{1}} \mathbb{E}\|x(t)\|^{2}<\infty$.

Let $\mathbb{Z}$ be the closed subspace of all continuous process $x$ that belong to the space $C\left(J_{1}, \mathcal{L}_{2}(\Omega ; H)\right)$ consisting of $\mathfrak{F}_{t}$ adapted measurable process and $\mathfrak{F}_{0}$-adapted processes $\phi, \xi \in \mathcal{L}_{2}(\Omega, \mathcal{B})$ and the restriction $x: J \rightarrow \mathcal{L}_{2}(\Omega, \mathcal{B})$ is continuous. Let $\|\cdot\|_{\mathbb{Z}}$ be a seminorm in $\mathbb{Z}$ defined by

$$
\|x\|_{\mathbb{Z}}^{2}=\sup _{t \in J}\left\|x_{t}\right\|_{\mathcal{B}}^{2}
$$

where

$$
\begin{gathered}
\left\|x_{t}\right\|_{\mathcal{B}}^{2} \leq \overline{K_{2}} \mathbb{E}\|\phi\|_{\mathcal{B}}^{2}+\overline{K_{3}} \sup _{0 \leq s \leq b}\left\{\mathbb{E}\|x(s)\|^{2}\right\}, \\
\overline{K_{2}}=\sup _{t \in J}\left\{K_{2}(t)\right\} \text { and } \overline{K_{3}}=\sup _{t \in J}\left\{K_{3}(t)\right\} \text {,It is easy to verify }
\end{gathered}
$$
that $\mathbb{Z}$ furnished with the norm topology as defined above is a Banach space.

Let $B(H)$ be the space of all bounded linear operators on $H$. Let $I$ be the identity operator on $H$. If $A$ is a linear operator on $H$, then $R(\lambda, A)=(\lambda I-A)^{-1}$ denotes the resolvent operator of $A$. We can use the notation

$$
k_{\beta}(t)=\frac{t^{\beta-1}}{\Gamma(\beta)}, t>0, \beta>0,
$$

where $\Gamma(\beta)$ is the Gamma function. If $\beta=0$, we set $k_{0}(t)=\delta(t)$, the delta distribution.

The foiiowing important defitions $2.1-2.8$ are carried out from Kexue et al. [27].

Definition 2.1 The Riemann-Liouville fractional integral of order $q>0$ is defined by

$$
\mathcal{J}_{t}^{q} x(t)=\int_{0}^{t} k_{q}(t-s) x(s) d s
$$

where.$x(t) \in \mathcal{L}_{1}([0, b] ; H)$.

Definition 2.2 The Riemann-Liouville fractional derivative of order $1<q \leq 2$ is defined by

$$
D_{t}^{q} x(t)=\frac{d^{2}}{d t^{2}} \mathcal{J}_{t}^{2-q} x(t)
$$

where $x(t) \in \mathcal{L}_{1}([0, b] ; H)$,

Definition 2.3 The Caputo fractional derivative of order $1<q \leq 2$ is defined by

$$
{ }^{c} D_{t}^{q} x(t)=D_{t}^{q}\left(x(t)-x(0)-x^{\prime}(0) t\right),
$$

where $\quad x(t) \in \mathcal{L}_{1}([0, b] ; H) \cap C^{1}([0, b] ; H), D_{t}^{q} x(t) \in$ $\mathcal{L}_{1}([0, b] ; H)$.

The Laplace transform for the Riemann-Liouville fractional integral is given by

$$
L\left[\mathcal{J}_{t}^{q} x(t)\right]=\frac{1}{\lambda^{q}} x_{L}(\lambda),
$$

where $x_{L}(\lambda)$ is the Laplace transform of $x$ given by

$$
x_{L}(\lambda)=\int_{0}^{\infty} e^{-\lambda t} x(t) d t, \operatorname{Re} \lambda>\omega_{0} .
$$

The Laplace transform of the Caputo derivative is given by

$$
L\left[{ }^{c} D_{t}^{q} x(t)\right]=\lambda^{q} x_{L}(\lambda)-x(0) \lambda^{q-1}-x^{\prime}(0) \lambda^{q-2}
$$


Consider a class of fractional differential system with infinite delay

$$
\begin{gathered}
{ }^{c} D_{t}^{q} x(t)=A x(t)+B u(t), t \in[0, b], \\
x(0)=x_{0} \in H, x^{\prime}(0)=x_{1} \in H,
\end{gathered}
$$

where $q \in(1,2],{ }^{c} D_{t}^{q}$ is the Caputo fractional derivative, $A$ is the infinitesimal generator of a strongly continuous $-q$-order cosine family $\left\{C_{q}(t)\right\}_{t \geq 0}$ on the Hilbert space $H$, the state values $x(\cdot)$ takes values in $H$, the control function $u(\cdot)$ is given in $\mathcal{L}_{2}(J, U)$ of admissible control functions with $U$, a Hilbert space, $B$ is a bounded linear operator form $U$ into. $H$.

Theorem 2.4 (Schauder fixed point theorem) Let $X$ be a real Banach space, $\mathbb{X} \subset X$ a nonempty closed bounded convex subset and $f: \mathbb{X} \rightarrow \mathbb{X}$ is compact. Then $f$ has a fixed point.

Theorem 2.5 (Lebesgue's dominated convergence theorem) Suppose $f_{n}: \mathbb{R} \rightarrow[-\infty, \infty]$ are (Lebesgue) measurable functions such that the pointwise limit $f(x)=\lim _{n \rightarrow \infty} f_{n}(x)$ exists. Assume there is an integrable $g: \mathbb{R} \rightarrow[0, \infty]$ with $\left|f_{n}(x)\right| \leq g(x)$ for each $x \in \mathbb{R}$. Then $f$ is integrable as is $f_{n}$ for each $n$, and

$$
\lim _{n \rightarrow \infty} \int_{\mathbb{R}} f_{n} d \mu=\int_{\mathbb{R}} \lim _{n \rightarrow \infty} f_{n} d \mu=\int_{\mathbb{R}} f d \mu .
$$

Definition 2.6 Let $q \in(1,2]$. A family $\left\{C_{q}(t)\right\}_{t \geq 0} \subset \mathcal{B}(H)$ is called a solution operator (or a strongly continuous $q$-order fractional cosine family) for the above problem (2) if the following conditions are satisfied

(a) $C_{q}(0)=I, I$ is the identity operator in $H$,

(b) $C_{q}(t) x$ is strongly continuous for $t \geq 0$, for every $x \in H$,

(c) $C_{q}(t) D(A) \subset D(A)$ and $A C_{q}(t) x_{0}=C_{q}(t) A x_{0}$ for all $x_{0} \in D(A), t \geq 0$,

(d) $C_{q}(t) x_{0}$ is a solution of $x(t)=x_{0}+\int_{0}^{t} k_{q}(t-$ $s) A x(s) d s$ for all $x_{0} \in D(A), t \geq 0$,

$\mathrm{A}$ is called the infinitesimal generator of . The strongly continuous $q$-order fractional cosine family is also called $q$-order cosine family.

The corresponding fractional sine family $\mathrm{S}_{q}: \mathbb{R}_{+} \rightarrow \mathcal{B}(H)$ associated with $C_{q}$ is defined by $S_{q}(t) x=\int_{0}^{t} ? C_{q}(s) x d s$ for, $t \geq 0, . x \in H$,

Definition 2.7 The fractional Riemann-Liouville family $\mathrm{T}_{q}: \mathbb{R}_{+} \rightarrow \mathcal{B}(H)$ associated with $C_{q}$ is defined by

$$
T_{q}(t)=\mathcal{J}_{t}^{q-1} C_{q}(t)
$$

Definition 2.8 The $q$-order cosine family $C_{q}$ is called exponentially bounded if there exist constants $M \geq 1$ and $\omega_{0} \geq 0$ such that

$$
\left\|C_{q}(t)\right\| \leq M e^{\omega_{0} t}, t \geq 0 .
$$

An operator $A$ is said to belong to $C^{q}\left(M, \omega_{0}\right)$, if problem (2) has an q-order cosine family $C_{q}(t)$ satisfying the above inequality.

Assume $A \in C^{q}\left(M, \omega_{0}\right)$ and let $C_{q}(t)$ be the corresponding $q$-order cosine family. Then we have

$$
\begin{aligned}
& \lambda^{q-1} R\left(\lambda^{q}, A\right) x_{0}=\int_{0}^{\infty} e^{-\lambda t} C_{q}(t) x_{0} d t, \operatorname{Re} \lambda>\omega_{0}, x_{0} \in H, \\
& \lambda^{q-2} R\left(\lambda^{q}, A\right) x_{0}=\int_{0}^{\infty} e^{-\lambda t} S_{q}(t) x_{0} d t, \operatorname{Re} \lambda>\omega_{0}, x_{0} \in H, \\
& R\left(\lambda^{q}, A\right) x_{0}=\int_{0}^{\infty} e^{-\lambda t} T_{q}(t) x_{0} d t, \operatorname{Re} \lambda>\omega_{0}, x_{0} \in H
\end{aligned}
$$

Based on the result found in Kexue et al. [27], the functixon $x \in \mathrm{C}([0, b] ; H)$ is called a mild solution of (2) if $\mathrm{x}$ satisfies

$$
x(t)=C_{q}(t) x_{0}+S_{q}(t) x_{1}+\int_{0}^{t} T_{q}(t-s) B u(s) d s, t \in J .
$$

The solution of system (1) for a given control $u(\cdot) \in$ $\mathcal{L}_{2}(J, U)$ denoted by $x(\cdot ; u)$. In particular, the state of system (1) at $t=b, x(b ; u)$ is called the terminal state with control $u$. The set $R_{b}(N)=\left\{x(b ; u) ; u(\cdot) \in \mathcal{L}_{2}(J, U)\right\}$ is called the reachable set of system (1). In what follows, $\overline{R_{b}(N)}$ stands for the closure of $R_{b}(N)$ in the space $H$.

Definition 2.9 The system (1) is said to be approximately controllable on the interval $J$ if $R_{b}(N)$ is dense in $H$, i.e., $\overline{R_{b}(N)}=H$.

In order to study the approximate controllability (see [12, $32,38,42,51])$ of the fractional control system (1), we need to introduce the relevant operator

$$
\begin{gathered}
\psi_{0}^{b}=\int_{0}^{b} T_{q}(b-s) B B^{*} T_{q}^{*}(b-s) d s, \\
R\left(\epsilon, \psi_{0}^{b}\right)=\left(\epsilon I+\psi_{0}^{b}\right)^{-1} \text { for } \epsilon>0,
\end{gathered}
$$

where $B^{*}$ denotes the adjoint of $B$ and $T_{q}^{*}(T)$ is the adjoint of $T_{q}(t)$. It is clear that the operator $\psi_{0}^{b}$ is a linear bounded operator.

(H0) $\epsilon R\left(\epsilon, \psi_{0}^{b}\right) \rightarrow 0$ as $\epsilon \rightarrow 0^{+}$in the strong operator topology.

Note that the assumption ( $\mathrm{H} 0$ ) is equivalent to the fact that the fractional linear control system (2) is approximately controllable on $J$.

Definition 2.10 It is clear that under these conditions the system (1) admits a mild solution $x(\cdot) \in P C\left(J, \mathcal{L}_{2}\right)$ if

(i) $x(t)$ is $\mathfrak{F}$-adapted for each $0 \leq t \leq b$,

(ii) $\int_{0}^{b} \mathbb{E}\|x(s)\|^{2} d s<\infty$, a.s.

(iii) For each $u \in \mathcal{L}_{2}^{\mathfrak{F}}(J, U)$ and $x(\cdot) \in \operatorname{PC}\left(J, \mathcal{L}_{2}\right)$ the following stochastic integral equation is satisfied

$$
\begin{gathered}
\mathrm{x}(t)=C_{q}(t)\left(\phi(0)+\mathrm{m}\left(x_{t_{1}}, \ldots, x_{t_{m}}\right)(0)\right)+S_{q}(t) \xi+\int_{0}^{t} T_{q}(t-s) f\left(s, x_{\rho\left(s, x_{s}\right)}\right) d s+\int_{0}^{t} T_{q}(t-s) B u(s) d s \\
+\int_{0}^{t} T_{q}(t-s)\left[\int_{-\infty}^{s} g\left(s, x_{\rho\left(s, x_{s}\right)}\right) d \omega(s)\right] d s+\int_{0}^{t} \int_{Z} T_{q}(t-s) \mathfrak{h}\left(s, x_{\rho\left(s, x_{s}\right)}, \eta\right) \widetilde{N}(d s, d \eta) \\
+\sum_{0<t_{k}<t} T_{q}\left(t-t_{k}\right) I_{k}\left(x_{t_{k}}\right)+\sum_{0<t_{k}<t} T_{q}(t-) \bar{I}_{k}\left(x_{t_{k}}\right), k=\overline{1, n} .
\end{gathered}
$$


(iv) $x_{0}(t)=\phi(t)+\mathbf{m}\left(x_{t_{1}}, \ldots, x_{t_{m}}\right)(t), x^{\prime}(0)=\xi$,

where

$$
\begin{gathered}
u(t)=B^{*} T_{q}^{*}(b-t) R\left(\epsilon, \psi_{0}^{b}\right)\left\{x_{b}-C_{q}(t)\left(\phi(0)+\mathrm{m}\left(x_{t_{1}}, \ldots, x_{t_{m}}\right)(0)\right)-S_{q}(t) \xi-\int_{0}^{t} T_{q}(b-s) f\left(s, x_{\rho\left(s, x_{s}\right)}\right) d s\right. \\
-\int_{0}^{t} T_{q}(b s)\left[\int_{-\infty}^{s} g\left(s, x_{\rho\left(s, x_{s}\right)}\right) d \omega(s)\right] d s-\int_{0}^{t} \int_{Z} T_{q}(b-s) \mathfrak{h}\left(s, x_{\rho\left(s, x_{s}\right)}, \eta\right) \widetilde{N}(d s, d \eta) \\
-\sum_{0<t_{k}<t} T_{q}\left(\mathrm{~b}-t_{k}\right) I_{k}\left(x_{t_{k}}\right)-\sum_{0<t_{k}<t} T_{q}(\mathrm{~b}-) \bar{I}_{k}\left(x_{t_{k}}\right), k=\overline{1, n}
\end{gathered}
$$

In order to prove the theorems, we need the following hypotheses:

(H1) The bounded linear operator $T_{q}(t)$ is compact for $t>0$ and there exists a positive constant $M$ such that

$$
\sup _{t \in J}\left\|T_{q}(t)\right\| \leq M .
$$

(H2) $A$ is an infinitesimal operator of a strongly continuous $q$-order cosine family $\left\{C_{q}(t): t \geq 0\right\}$ on $H$. Also $\left\|C_{q}(t)\right\| \leq$ $M_{C}$ and $\left\|S_{q}(t)\right\| \leq M_{S}$. Moreover the operators $C_{q}(t)$ and $S_{q}(t)$ are compact for $t>0$,

(H3) The functions $\mathbf{m}: H^{m} \rightarrow H$ is continuous, satisfy the Lipschitz condition and there exists a positive constant $G$ such that

$$
\mathbb{E}\left\|\mathbf{m}\left(x_{t_{1}}, \ldots, x_{t_{m}}\right)\right\|_{H}^{2} \leq G,
$$

for all,$\left(x_{t_{1}}, \ldots, x_{t_{m}}\right) \in H^{m}$,

(H4) $B$ is a bounded linear operator from $U$ into $H$, such that $\|B\|=N$, for a constant $N \geq 0$.

(H5) For each $\varphi \in \mathcal{B}, K_{g}(t)=\lim _{a \rightarrow \infty} \int_{-a}^{0} g(s, \varphi) d \omega(s)$ exists and is continuous. Also there exists a positive constant $M_{g}$ such that. $\mathbb{E}\left\|K_{g}(t)\right\|_{H}^{2} \leq M_{g}$,

(H6) The non-linear function $\mathfrak{h}$ is a Borel measurable function which satisfy the Lipschitz continuity condition and there exists positive constants $M_{\mathfrak{h}}>0$ and $L_{\mathfrak{h}}>0$ such that

$$
\int_{Z}\|\mathfrak{h}(t, x, \eta)\|_{H}^{2} \lambda(d \eta) d s \leq M_{\mathfrak{h}}\left(1+\|x\|_{\mathcal{B}}^{2}\right),
$$

$\int_{Z}\|\mathfrak{h}(t, x, \eta)\|_{H}^{4} \lambda(d \eta) \leq L_{\mathfrak{h}}\left(1+\|x\|_{\mathcal{B}}^{4}\right)$, for all $x \in \mathcal{B}, t \in J$.

(H7) The functions $t \rightarrow \phi_{t}$ and $t \rightarrow \xi_{t}$ are well defined from $\mathcal{Z}\left(\rho^{-}\right)=\{\rho(s, \tau) ;(s, \tau) \in J \times \mathcal{B}, \rho(s, \tau) \leq 0\}$ into $\mathcal{B}$ and there exists a continuous and bounded functions $\mathcal{H}^{\phi}, \mathcal{H}^{\xi}: \mathcal{Z}\left(\rho^{-}\right) \rightarrow \mathbb{R}$ such that

$\left\|\phi_{t}\right\|_{\mathcal{B}}^{2} \leq \mathcal{H}^{\phi}(t)\|\phi\|_{\mathcal{B}}^{2}$ and $\left\|\xi_{t}\right\|_{\mathcal{B}}^{2} \leq \mathcal{H}^{\xi}(t)\|\xi\|_{\mathcal{B}}^{2}$ for every $t \in Z\left(\rho^{-}\right)$.

(H8) The functions $I_{k}, \bar{I}_{k}: H \rightarrow H$ are continuous and there exists constants $\hat{c}_{k}, \hat{d}_{k}, k=\overline{1, n}$ such that

$$
\mathbb{E}\left\|I_{k}(x)\right\|^{2} \leq \hat{c}_{k}\|x\|^{2}, \mathbb{E}\left\|\bar{I}_{k}(x)\right\|^{2} \leq \hat{d}_{k}\|x\|^{2},
$$

for every $x \in \mathcal{B}$ and $k=\overline{1, n}$,

(H9) The functions $f: J \times \mathcal{B} \rightarrow H, g: J \times \mathcal{B} \rightarrow \mathcal{L}_{Q}(K, H)$ and $\mathfrak{h}: J \times \mathcal{B} s \in J \times Z \rightarrow H$ satisfies the following conditions:

(i) Let $x:(-\infty, b] \rightarrow H$ be such that $x \in P C\left(J, \mathcal{L}_{2}\right)$. The functtions $\quad t \rightarrow f\left(s, x_{\rho\left(t, x_{t}\right)}\right), t \rightarrow g\left(s, x_{\rho\left(t, x_{t}\right)}\right)$ and $t \rightarrow$ $\mathfrak{h}\left(s, x_{\rho\left(s, x_{s}\right)}, \eta\right)$ are measurable on $J$. Also the functions $t \rightarrow f\left(s, x_{\rho\left(t, x_{t}\right)}\right), t \rightarrow g\left(s, x_{\rho\left(t, x_{t}\right)}\right)$ and $t \rightarrow \mathfrak{h}\left(s, x_{\rho\left(s, x_{s}\right)}, \eta\right)$ are continuous on $Z\left(\rho^{-}\right) \cup J$ for every $s \in J$.

(ii) For $\varepsilon>0$, there exists a function $W_{\varepsilon} \in \mathcal{L}_{1}\left(J, \mathbb{R}^{+}\right)$such that $\sup _{\|\varphi\|^{2} \leq \varepsilon} \mathbb{E}\|f(t, \varphi)\|^{2} \leq W_{\varepsilon}(t)$ for a.e $t \in J$ and $\liminf _{\varepsilon \rightarrow \infty} \int_{0}^{b} \frac{W_{\varepsilon}(t)}{\varepsilon}=\delta_{0}<\infty$.

(iii) For $\varepsilon>0$, there exists a function $\Omega_{\varepsilon} \in \mathcal{L}_{1}\left(J, \mathbb{R}^{+}\right)$ such that $\sup _{\|\varphi\|^{2} \leq \varepsilon} \mathbb{E}\|g(t, \varphi)\|_{Q}^{2} \leq \Omega_{\varepsilon}(t)$ for a.e $t \in J$ and $\liminf _{\varepsilon \rightarrow \infty} \int_{0}^{b} \frac{\Omega_{\varepsilon}(t)}{\varepsilon}=\delta_{1}<\infty$.

(iv) For $\varepsilon>0$, there exists a function $\Theta_{\varepsilon} \in \mathcal{L}_{1}\left(J, \mathbb{R}^{+}\right)$ such that $\sup _{\mathbb{E}}\|\mathfrak{h}(t, \varphi, \eta)\|_{Q}^{2} \leq \Theta_{\varepsilon}(t)$ for a.e $t \in J$ and $\liminf _{\varepsilon \rightarrow \infty} \int_{0}^{b} \frac{\|\varphi\|^{2} \leq \varepsilon}{\varepsilon}=\delta_{2}<\infty$

We can prove the next lemma using the phase space axioms.

Lemma 2.11 Let $x:(-\infty, b] \rightarrow H$ be a function such that $x \in P C\left(J, \mathcal{L}_{2}\right)$.

Then

$\mathbb{E}\left\|x_{s}\right\|_{\mathcal{B}}^{2} \leq\left(\mathcal{H}_{2}+\mathfrak{H}_{1}\right)\|\phi\|_{\mathcal{B}}^{2}+\mathcal{H}_{3} \sup \left\{\mathbb{E}\|x(\theta)\|^{2} ; \theta\right.$ $\in[0, \max \{0, s\}]\}, s \in Z\left(\rho^{-}\right) \cup J$,

and $\mathbb{E}\left\|x_{s}\right\|_{\mathcal{B}}^{2} \leq\left(\mathcal{H}_{2}+\mathfrak{H}_{2}\right)\|\xi\|_{\mathcal{B}}^{2}+\mathcal{H}_{3} \sup \left\{\mathbb{E}\|x(\theta)\|^{2} ; \theta \in\right.$ $[0, \max \{0, s\}]\}, s \in \mathcal{Z}\left(\rho^{-}\right) \cup J$, where

$$
\begin{aligned}
\mathfrak{H}_{1} & =\sup _{t \in Z\left(\rho^{-}\right)} H^{\phi}(t), \mathfrak{H}_{2}=\sup _{t \in Z\left(\rho^{-}\right)} H^{\xi}(t), \mathcal{H}_{2}=\sup _{t \in J} \overline{K_{2}}(t), \\
\mathcal{H}_{3} & =\sup _{t \in J} \overline{K_{3}}(t) .
\end{aligned}
$$

\section{Approximate Controllability}

Theorem 3.1 Assume that the hypotheses $(H 1)-(H 9)$ are satisfied. Further, suppose that for all $\varepsilon>0$

$$
11 M^{2} \delta \mathcal{H}_{3}\left(1+\frac{11 M^{4} N^{4} b}{\epsilon^{2}}\right)\left(1+2 b^{2}\left[\operatorname{Tr}(Q)+\left(M_{\mathfrak{h}}+\sqrt{L_{\mathfrak{h}}}\right)\right]\right)<1,
$$

then the system (1) has a solution on $J$.

Proof. Let $\mathcal{B}_{b}^{0}=\left\{x \in P C\left(J, \mathcal{L}_{2}\right): x_{0} \in \mathcal{B}\right\}$ be the space endowed with uniform convergence topology. On the space $\mathcal{B}_{b}$, consider a set $\mathcal{B}_{r}=\left\{x \in \mathcal{B}_{b}^{0}:\|x\|^{2} \leq r\right\}$,

where $r$ is a positive constant. Let $\|\cdot\|_{b}$ be the semi-norm defined by

$$
\begin{gathered}
\|x\|_{b}^{2}=\sup _{t \in J}\left\|x_{t}\right\|_{\mathcal{B}}^{2} \\
\left\|x_{t}\right\|_{\mathcal{B}}^{2} \leq \overline{K_{2}} \mathbb{E}\|\phi\|_{\mathcal{B}_{b}}^{2}+\overline{K_{3}} \sup _{0 \leq s \leq b}\left\{\mathbb{E}\|x(s)\|^{2}\right\} .
\end{gathered}
$$

For $\varepsilon>0$, define the operator $\Upsilon: \mathcal{B}_{b}^{0} \rightarrow \mathcal{B}_{b}^{0}$ by $\Upsilon x(t)=$ $\tilde{z}(t)$, where 


$$
\begin{gathered}
\tilde{z}(t)=C_{q}(t)\left(\phi(0)+\mathbf{m}\left(x_{t_{1}}, \ldots, x_{t_{m}}\right)(0)\right)+S_{q}(t) \xi+\int_{0}^{t} T_{q}(t-s) f\left(s, x_{\rho\left(s, x_{s}\right)}\right) d s+\int_{0}^{t} T_{q}(t-s) B u(s) d s \\
+\int_{0}^{t} T_{q}(t-s)\left[\int_{-\infty}^{s} g\left(s, x_{\rho\left(s, x_{s}\right)}\right) d \omega(s)\right] d s+\int_{0}^{t} \int_{Z} T_{q}(t-s) \mathfrak{h}\left(s, x_{\rho\left(s, x_{s}\right)}, \eta\right) \widetilde{N}(d s, d \eta) \\
+\sum_{0<t_{k}<t} T_{q}\left(t-t_{k}\right) I_{k}\left(x_{t_{k}}\right)+\sum_{0<t_{k}<t} T_{q}\left(t-t_{k}\right) \bar{I}_{k}\left(x_{t_{k}}\right), \\
k=\overline{1, n}
\end{gathered}
$$

and

$$
\begin{gathered}
\tilde{v}(t)=B^{*} T_{q}^{*}(b-t) R\left(\epsilon, Y_{0}^{b}\right)\left\{x_{b}-C_{q}(t)\left(\phi(0)+\mathrm{m}\left(x_{t_{1}}, \ldots, x_{t_{m}}\right)(0)\right)-S_{q}(t) \xi-\int_{0}^{t} T_{q}(\mathrm{~b}-s) f\left(s, x_{\rho\left(s, x_{s}\right.}\right) d s\right. \\
-\int_{0}^{t} T_{q}(b-s)\left[\int_{-\infty}^{s} g\left(s, x_{\rho\left(s, x_{s}\right)}\right) d \omega(s)\right] d s-\int_{0}^{t} \int_{Z} T_{q}(b-s) \mathfrak{h}\left(s, x_{\rho\left(s, x_{s}\right)}, \eta\right) \widetilde{N}(d s, d \eta) \\
\left.\left.-\sum_{0<t_{k}<t} T_{q}\left(\mathrm{~b}-t_{k}\right) I_{k}\left(x_{t_{k}}\right)-\sum_{0<t_{k}<t} T_{q}\left(b-t_{k}\right) \bar{I}_{k}\left(x_{t_{k}}\right)\right)\right\}, k=\overline{1, n}
\end{gathered}
$$

we shall show that the operator $\Upsilon: \mathcal{B}_{b}^{0} \rightarrow \mathcal{B}_{b}^{0}$ by $\Upsilon x(t)=\tilde{z}(t)$ has a fixed point, for all $\varepsilon>0$. For that, we have to prove for $\varepsilon>0$ there exists an $r>0$ such that $\Upsilon\left(\mathcal{B}_{r}\right) \subset \mathcal{B}_{r}$. Suppose $r$, there exists a function $x(\cdot) \in \mathcal{B}_{r}$, but $\Upsilon(x) \notin \mathcal{B}_{r}$ that is $\mathbb{E} \|(\Upsilon x)(t)) \|_{H}^{2}>r$.

For any $x \in \mathcal{B}_{r}$, it follows from lemma (2.11) that let us assume that $\Upsilon\left(\mathcal{B}_{r}\right) \& \mathcal{B}_{r}$. Then for each positive number

$$
\mathbb{E}\left\|\bar{x}_{\rho\left(s, \bar{x}_{s}\right)}\right\|_{\mathcal{B}}^{2} \leq\left(\mathcal{H}_{2}+\mathfrak{h}_{1}\right)\|\phi\|_{\mathcal{B}}^{2}+\mathcal{H}_{3} r \vee\left(\mathcal{H}_{2}+\mathfrak{H}_{2}\right)\|\xi\|_{\mathcal{B}}^{2}+\mathcal{H}_{3} r=r^{*}
$$

For, $\varepsilon>0$,

$$
\begin{gathered}
\mathbb{E}\|\tilde{v}(t)\|^{2}=\mathbb{E} \| B^{*} T_{q}^{*}(b-t) R\left(\epsilon, Y_{0}^{b}\right)\left\{x_{b}-C_{q}(t)\left(\phi(0)+\mathrm{m}\left(x_{t_{1}}, \ldots, x_{t_{m}}\right)(0)\right)-S_{q}(t) \xi\right. \\
-\int_{0}^{t} T_{q}(b-s) f\left(s, x_{\rho\left(s, x_{s}\right)}-\int_{0}^{t} T_{q}(b-s)\left[\int_{-\infty}^{s} g\left(s, x_{\rho\left(s, x_{s}\right)}\right) d \omega(s)\right] d s-\int_{0}^{t} \int_{Z} T_{q}(b-s) \mathfrak{h}\left(s, x_{\rho\left(s, x_{s}\right)}, \eta\right) \widetilde{N}(d s, d \eta)\right. \\
\left.\left.-\sum_{0<t_{k}<t} T_{q}\left(\mathrm{~b}-t_{k}\right) I_{k}\left(x_{t_{k}}\right)-\sum_{0<t_{k}<t} T_{q}\left(b-t_{k}\right) \bar{I}_{k}\left(x_{t_{k}}\right)\right)\right\}, k=\overline{1, n}, \\
\leq \frac{11 M^{2} N^{2}}{\epsilon^{2}}\left\{\left\|x_{b}\right\|^{2}+M_{C}^{2}\left[\|\phi\|_{\mathcal{B}}^{2}+M_{\mathbf{m}}\right]+M_{S}^{2}\|\xi\|_{\mathcal{B}}^{2}+M^{2} \int_{0}^{b} W_{r^{*}}(s) d s+M^{2} b^{2}\left[2 M_{g}+2 \operatorname{Tr}(Q) \int_{0}^{b} \Omega_{r^{*}}(s) d s\right]\right. \\
\left.+M^{2} b^{2}\left(2 M_{\mathfrak{h}}+2 \sqrt{L_{\mathfrak{h}}}\right) \int_{0}^{b} \Theta_{r^{*}}(s) d s+M^{2} \sum_{k=1}^{m}\left(\hat{c}_{k}+\hat{d}_{k}\right)\right\}, k=\overline{1, n}, \\
\leq \frac{11 M^{2} N^{2}}{\epsilon^{2}}\left\{\left\|x_{b}\right\|^{2}+M_{C}^{2}\left[\|\phi\|_{\mathcal{B}}^{2}+M_{\mathbf{m}}\right]+M_{S}^{2}\|\xi\|_{\mathcal{B}}^{2}+M^{2} \int_{0}^{b} W_{r^{*}}(s) d s\right. \\
\left.+M^{2} b^{2}\left[2 M_{g}+2 \operatorname{2Tr}(Q) \int_{0}^{b} \Omega_{r^{*}}(s) d s\right]+M^{2} b^{2}\left(2 M_{\mathfrak{h}}+2 \sqrt{L_{\mathfrak{h}}}\right) \int_{0}^{b} \Theta_{r^{*}}(s) d s+\sum_{k=1}^{m}\left(\hat{c}_{k}+\hat{d}_{k}\right)\right\}, k=\overline{1, n},
\end{gathered}
$$

where

$$
M_{\mathbf{m}}=\|\phi\|_{\mathcal{B}}^{2}+\left\|m_{1}\left(x_{t_{1}}, \ldots, x_{t_{m}}\right)(t)\right\|_{H}^{2}+\|\xi\|_{\mathcal{B}}^{2}
$$

For $\varepsilon>0$ and $r>0$, we have

$$
\begin{gathered}
r<\mathbb{E}\|\Upsilon x(t)\|^{2}=\mathbb{E} \| C_{q}(t)\left(\phi(0)+\mathbf{m}\left(x_{t_{1}}, \ldots, x_{t_{m}}\right)(0)\right)+S_{q}(t) \xi+\int_{0}^{t} T_{q}(t-s) f\left(s, x_{\rho\left(s, x_{s}\right)}\right) d s+\int_{0}^{t} T_{q}(t-s) B u(s) d s \\
+\int_{0}^{t} T_{q}(t-s)\left[\int_{-\infty}^{s} g\left(s, x_{\rho\left(s, x_{s}\right)}\right) d \omega(s)\right] d s+\int_{0}^{t} \int_{Z} T_{q}(t-s) \mathfrak{h}\left(s, x_{\rho\left(s, x_{s}\right)}, \eta\right) \widetilde{N}(d s, d \eta) \\
+\sum_{0<t_{k}<t} T_{q}\left(t-t_{k}\right) I_{k}\left(x_{t_{k}}\right)+\sum_{0<t_{k}<t} T_{q}\left(t-t_{k}\right) \bar{I}_{k}\left(x_{t_{k}}\right) \|^{2}, k=\overline{1, n} . \\
\leq 11 M_{C}^{2}\left[\|\phi\|_{\mathcal{B}}^{2}+M_{\mathbf{m}}\right]+11 M_{S}^{2}\|\xi\|_{\mathcal{B}}^{2}+11 M^{2} \int_{0}^{b} W_{r^{*}}(s) d s \\
+\frac{121 M^{4} N^{4} b}{\epsilon^{2}}\left\{\left\|x_{b}\right\|^{2}+M_{C}^{2}\left[\|\phi\|_{\mathcal{B}}^{2}+M_{\mathbf{m}}\right]+M_{S}^{2}\|\xi\|_{\mathcal{B}}^{2}+M^{2} \int_{0}^{b} W_{r^{*}}(s) d s\right.
\end{gathered}
$$




$$
\begin{gathered}
+M^{2} b^{2}\left[2 M_{g}+2 \operatorname{Tr}(Q) \int_{0}^{b} \Omega_{r^{*}}(s) d s\right]+M^{2} b^{2}\left(2 M_{\mathfrak{\natural}}+2 \sqrt{L_{\mathfrak{h}}}\right) \int_{0}^{b} \Theta_{r^{*}}(s) d s \\
\left.+M^{2} \sum_{k=1}^{m}\left(\hat{c}_{k}+\hat{d}_{k}\right)\right\}+11 M^{2} b^{2}\left[2 M_{g}+2 \operatorname{Tr}(Q) \int_{0}^{b} \Omega_{r^{*}}(s) d s\right] \\
+11 M^{2} b^{2}\left(2 M_{\mathfrak{h}}+2 \sqrt{L_{\mathfrak{h}}}\right) \int_{0}^{b} \Theta_{r^{*}}(s) d s+11 M^{2} \sum_{k=1}^{m}\left(\hat{c}_{k}+\hat{d}_{k}\right) \\
\leq K^{*}+\frac{121 M^{6} N^{4} b}{\epsilon^{2}}\left\{\int_{0}^{b} W_{r^{*}}(s) d s+2 b^{2}\left[\operatorname{Tr}(Q) \int_{0}^{b} \Omega_{r^{*}}(s) d s+\left(M_{\mathfrak{h}}+\sqrt{L_{\mathfrak{h}}}\right) \int_{0}^{b} \Theta_{r^{*}}(s) d s\right]\right\} \\
+11 M^{2}\left\{\int_{0}^{b} W_{r^{*}}(s) d s+2 b^{2}\left[\operatorname{Tr}(Q) \int_{0}^{b} \Omega_{r^{*}}(s) d s+\left(M_{\mathfrak{h}}+\sqrt{L_{\mathfrak{h}}}\right) \int_{0}^{b} \Theta_{r^{*}}(s) d s\right]\right\}
\end{gathered}
$$

Note that $K^{*}$ is independent of $r$ and $r^{*} \rightarrow \infty$ as $r \rightarrow \infty$. Now

$$
\begin{aligned}
& \liminf _{r \rightarrow \infty} \int_{0}^{b} \frac{W_{r^{*}}(s)}{r} d s=\liminf _{r \rightarrow \infty} \int_{0}^{b}\left\{\frac{W_{r^{*}}(s)}{r^{*}} \frac{r^{*}}{r}\right\} d s=\delta_{0} \mathcal{H}_{3}, \\
& \liminf _{r \rightarrow \infty} \int_{0}^{b} \frac{\Omega_{r^{*}(s)}}{r} d s=\liminf _{r \rightarrow \infty} \int_{0}^{b}\left\{\frac{\Omega_{r^{*}}(s)}{r^{*}} \frac{r^{*}}{r}\right\} d s=\delta_{1} \mathcal{H}_{3}, \\
& \liminf _{r \rightarrow \infty} \int_{0}^{b} \frac{\Theta_{r^{*}(s)}}{r} d s=\liminf _{r \rightarrow \infty} \int_{0}^{b}\left\{\frac{\Theta_{r^{*}(s)}}{r^{*}} \frac{r^{*}}{r}\right\} d s=\delta_{2} \mathcal{H}_{3},
\end{aligned}
$$

Hence. $\delta=\max \left\{\delta_{0}, \delta_{1}, \delta_{2}\right\}$. We have for $\varepsilon>0$,

$$
11 M^{2} \delta \mathcal{H}_{3}\left(1+\frac{11 M^{4} N^{4} b}{\epsilon^{2}}\right)\left(1+2 b^{2}\left[\operatorname{Tr}(Q)+\left(M_{\mathfrak{h}}+\sqrt{L_{\mathfrak{h}}}\right)\right]\right) \geq 1,
$$

which is contradictive to our assumption. Hence for $\varepsilon>0$, there exist $r>0$ such that $\Upsilon\left(\mathcal{B}_{r}\right) \subset \mathcal{B}_{r}$.

Next we shall prove that arbitrary $\varepsilon>0$, the operator $\Upsilon$ maps $\mathcal{B}_{r}$ into a relatively compact subset of $\mathcal{B}_{r}$. First, we prove that the set $\mathrm{V}(t)=\left\{\Upsilon x(t) ; x \in \mathcal{B}_{r}\right\}$ is relatively compact in $H$ for every $t \in J$. It is clear that the case $t=0$ is obvious. For $0<\epsilon<t \leq b$, define $\left(\Upsilon^{\epsilon} x\right)(t)=T_{q}(\epsilon) \tilde{z}(t-\varepsilon)$, since $T_{q}(t)$ is compact and $\tilde{z}(t-\epsilon)$ is bounded on $\mathcal{B}_{r}$, the set $\mathrm{V}_{\epsilon}(t)=$ $\left\{\left(\Upsilon^{\epsilon} x\right)(t) ; x(\cdot) \in \mathcal{B}_{r}\right\}$ is relatively compact in $H$. That is, a finite set $\left\{y_{i}, 1 \leq i \leq n\right\}$ in $H$ exists such that $\mathrm{V}_{\epsilon}(t) \subset$ $\bigcup_{i=1}^{n} \widetilde{N}\left(y_{i}, \frac{\tau}{2}\right)$, where $\breve{N}\left(y_{i}, \frac{\tau}{2}\right)$ is an open ball in $H$ with center at $y_{i}$ and radius $\frac{\tau}{2}$. On the other hand,

$$
\begin{gathered}
\mathbb{E}\left\|(\Upsilon x)(t)-\left(\Upsilon^{\epsilon} x\right)(t)\right\|^{2} \leq \frac{77 M^{4} N^{4} b}{\epsilon^{2}}\left\{\left\|x_{b}\right\|^{2}+M_{C}^{2}\left[\|\phi\|_{\mathcal{B}}^{2}+M_{\mathbf{m}}\right]+M_{S}^{2}\|\xi\|_{\mathcal{B}}^{2}\right. \\
\left.+M^{2} \int_{0}^{b} W_{r^{*}}(s) d s+M^{2} b^{2}\left[2 M_{g}+2 \operatorname{Tr}(Q) \int_{t-\varepsilon}^{t} \Omega_{r^{*}}(s) d s\right]+M^{2} b^{2}\left(2 M_{\mathfrak{\zeta}}+2 \sqrt{L_{\mathfrak{h}}}\right) \int_{0}^{b} \Theta_{r^{*}}(s) d s+M^{2} \sum_{k=1}^{m}\left(\hat{c}_{k}+\hat{d}_{k}\right)\right\} \\
+7 M^{2} \int_{t-\varepsilon}^{t} W_{r^{*}}(s) d s+7 M^{2} b^{2}\left[2 M_{g}+2 \operatorname{Tr}(Q) \int_{t-\varepsilon}^{t} \Omega_{r^{*}}(s) d s\right]+7 M^{2} b^{2}\left(2 M_{\mathfrak{h}}+2 \sqrt{L_{\mathfrak{h}}}\right) \int_{t-\varepsilon}^{t} \Theta_{r^{*}}(s) d s \leq \frac{\tau}{2}
\end{gathered}
$$

Consequently $\mathrm{V}(t) \subset \bigcup_{i=1}^{n} \widetilde{N}\left(y_{i}, \tau\right)$. Hence for each $t \in J, \mathrm{~V}(t)$ is relatively compact in $H$.

Next we shall prove that $\mathrm{V}=\left\{(\Upsilon x)(\cdot): x(\cdot) \in \mathcal{B}_{r}\right\}$ is equicontinuous on $J$. For $0<t_{1}<t_{2} \leq b$,

$$
\begin{gathered}
\mathbb{E}\left\|\tilde{z}\left(t_{2}\right)-\tilde{z}\left(t_{1}\right)\right\|^{2}=\mathbb{E} \| C_{q}\left(t_{2}\right)\left(\phi(0)+\mathrm{m}\left(x_{t_{1}}, \ldots, x_{t_{m}}\right)(0)\right)+S_{q}\left(t_{2}\right) \xi+\int_{0}^{t_{2}} T_{q}\left(t_{2}-s\right) f\left(s, x_{\rho\left(s, x_{s}\right)}\right) d s \\
+\int_{0}^{t_{2}} T_{q}\left(t_{2}-s\right) B u(s) d s+\int_{0}^{t_{2}} T_{q}\left(t_{2} s\right)\left[\int_{-\infty}^{s} g\left(s, x_{\rho\left(s, x_{s}\right.}\right) d \omega(s)\right] d s \\
+\int_{0}^{t_{2}} \int_{Z} T_{q}\left(t_{2}-s\right) \mathfrak{h}\left(s, x_{\rho\left(s, x_{s}\right)}, \eta\right) \widetilde{N}(d s, d \eta)+\sum_{0<t_{k}<t} T_{q}\left(t_{2}-t_{k}\right) I_{k}\left(x_{t_{k}}\right) \\
\left.+\sum_{0<t_{k}<t} T_{q}\left(t_{2}-t_{k}\right) \bar{I}_{k}\left(x_{t_{k}}\right)\right\}-\left\{C_{q}\left(t_{1}\right)\left(\phi(0)+\mathrm{m}\left(x_{t_{1}}, \ldots, x_{t_{m}}\right)(0)\right)+S_{q}\left(t_{1}\right) \xi\right. \\
\left.\left.+\int_{0}^{t_{1}} \int_{Z} T_{q}\left(t_{2}-s\right) \mathfrak{h}\left(s, x_{\rho\left(s, x_{s}\right)}, \eta\right) \widetilde{N}(d s, d \eta)+\sum_{0<t_{k}<t} T_{q}\left(t_{1}-t_{k}\right) I_{k}\left(x_{t_{k}}\right)+\sum_{0<t_{k}<t} T_{q}\left(t-t_{k}\right) \bar{I}_{k}\left(x_{t_{k}}\right)\right\}\right\} \|^{2}, k=\overline{1, n}, \\
\leq 19\left\|C_{q}\left(t_{2}\right)-C_{q}\left(t_{1}\right)\right\|^{2}\left(\|\phi\|_{\mathcal{B}}^{2}+G\right)+19\left\|S_{q}\left(t_{2}\right)-S_{q}\left(t_{1}\right)\right\|^{2}\|\xi\|_{\mathcal{B}}^{2} \\
+19 M^{2} N^{2} \int_{t_{1}}^{t_{2}}\|v(s)\|^{2} d s+19 N^{2} \int_{0}^{t_{1}}\left\|T_{q}\left(t_{2}-s\right)-T_{q}\left(t_{1}-s\right)\right\|^{2}\|v(s)\|^{2} d s \\
+19 M^{2} \int_{t_{1}}^{t_{2}} W_{r^{*}}(s) d s+19 \int_{0}^{t_{1}}\left\|T_{q}\left(t_{2}-s\right)-T_{q}\left(t_{1}-s\right)\right\|^{2} W_{r^{*}}(s) d s
\end{gathered}
$$




$$
\begin{aligned}
& +19 M^{2}\left(2 M_{g}+2 \operatorname{Tr}(Q) \int_{t_{1}}^{t_{2}} \Omega_{r^{*}}(s) d s\right)+19\left(2 M_{g}+2 \operatorname{Tr}(Q) \int_{0}^{t_{1}}\left\|T_{q}\left(t_{2}-s\right)-T_{q}\left(t_{1}-s\right)\right\|^{2} \Omega_{r^{*}}(s) d s\right) \\
& +19 M^{2}\left(2 M_{\mathfrak{h}}+2 \sqrt{L_{\mathfrak{h}}}\right) \int_{t_{1}}^{t_{2}} \Theta_{r^{*}}(s) d s+19\left(2 M_{\mathfrak{h}}+2 \sqrt{L_{\mathfrak{h}}}\right) \int_{0}^{t_{1}}\left\|T_{q}\left(t_{2}-s\right)-T_{q}\left(t_{1}-s\right)\right\|^{2} \Theta_{r^{*}}(s) d s \\
& +19 M^{2} \sum_{t_{1}<t_{k}<t_{2}}\left\|I_{k}\left(\bar{x}_{t_{k}}\right)\right\|^{2}+19 \sum_{0<t_{k}<t_{1}}\left\|T_{q}\left(t_{2}-t_{k}\right)-T_{q}\left(t_{1}-t_{k}\right)\right\|^{2}\left\|I_{k}\left(\bar{x}_{t_{k}}\right)\right\|^{2} \\
& +19 M^{2} \sum_{t_{1}<t_{k}<t_{2}}\left\|\bar{I}_{k}\left(\bar{x}_{t_{k}}\right)\right\|^{2}+19 \sum_{0<t_{k}<t_{1}}\left\|T_{q}\left(t_{2}-t_{k}\right)-T_{q}\left(t_{1}-t_{k}\right)\right\|^{2}\left\|\bar{I}_{k}\left(\bar{x}_{t_{k}}\right)\right\|^{2} \\
& \leq 19\left\|C_{q}\left(t_{2}\right)-C_{q}\left(t_{1}\right)\right\|^{2}\left(\|\phi\|_{\mathcal{B}}^{2}+G\right)+19\left\|S_{q}\left(t_{2}\right)-S_{q}\left(t_{1}\right)\right\|^{2}\|\xi\|_{\mathcal{B}}^{2} \\
& +\frac{209 M^{4} N^{4}}{\epsilon^{2}} \int_{t_{1}}^{t_{2}}\left\{\left\|x_{b}\right\|^{2}+M^{2}\left(\|\phi\|_{\mathcal{B}}^{2}+G\right)+M^{2}\|\xi\|_{\mathcal{B}}^{2}+M^{2} \int_{0}^{b} W_{r^{*}}(s) d s\right. \\
& +M^{2}\left(2 M_{g}+2 \operatorname{Tr}(Q) \int_{0}^{b} \Omega_{r^{*}}(s) d s+M^{2}\left(2 M_{\mathfrak{h}}+2 \sqrt{L_{\mathfrak{h}}}\right) \int_{0}^{t_{1}} \Theta_{r^{*}}(s) d s+M^{2} \sum_{k=1}^{m}\left(\hat{c}_{k}+\hat{d}_{k}\right)\right\} \\
& +\frac{209 M^{2} N^{4}}{\epsilon^{2}} \int_{0}^{t_{1}}\left\|T\left(t_{2}-s\right)-T\left(t_{1}-s\right)\right\|^{2}\left\{\left\|x_{b}\right\|^{2}+M^{2}\left(\|\phi\|_{\mathcal{B}}^{2}+G\right)+M^{2}\|\xi\|_{\mathcal{B}}^{2}+M^{2} \int_{0}^{b} W_{r^{*}}(s) d s\right. \\
& +M^{2}\left(2 M_{g}+2 \operatorname{Tr}(Q) \int_{0}^{b} \Omega_{r^{*}}(s) d s+M^{2}\left(2 M_{\mathfrak{h}}+2 \sqrt{L_{\mathfrak{h}}}\right) \int_{0}^{t_{1}} \Theta_{r^{*}}(s) d s+M^{2} \sum_{k=1}^{m}\left(\hat{c}_{k}+\hat{d}_{k}\right)\right\} \\
& +19 M^{2} \int_{t_{1}}^{t_{2}} W_{r^{*}}(s) d s+19 \int_{0}^{t_{1}}\left\|T_{q}\left(t_{2}-s\right)-T_{q}\left(t_{1}-s\right)\right\|^{2} W_{r^{*}}(s) d s \\
& +19 M^{2}\left(2 M_{g}+2 \operatorname{Tr}(Q) \int_{t_{1}}^{t_{2}} \Omega_{r^{*}}(s) d s+19\left(2 M_{g}+2 \operatorname{Tr}(Q) \int_{0}^{t_{1}}\left\|T_{q}\left(t_{2}-s\right)-T_{q}\left(t_{1}-s\right)\right\|^{2} \Omega_{r^{*}}(s) d s\right.\right. \\
& +19 M^{2}\left(2 M_{\mathfrak{\zeta}}+2 \sqrt{L_{\mathfrak{\natural}}}\right) \int_{t_{1}}^{t_{2}} \Theta_{r^{*}}(s) d s+19 \int_{0}^{t_{1}}\left\|T_{q}\left(t_{2}-s\right)-T_{q}\left(t_{1}-s\right)\right\|^{2}\left(2 M_{\mathfrak{\zeta}}+2 \sqrt{L_{\mathfrak{\zeta}}}\right) \Theta_{r^{*}}(s) d s \\
& +19 M^{2} \sum_{t_{1}<t_{k}<t_{2}}\left\|I_{k}\left(\bar{x}_{t_{k}}\right)\right\|^{2}+19 \sum_{0<t_{k}<t_{1}}\left\|T_{q}\left(t_{2}-t_{k}\right)-T_{q}\left(t_{1}-t_{k}\right)\right\|^{2}\left\|I_{k}\left(\bar{x}_{t_{k}}\right)\right\|^{2} \\
& +19 M^{2} \sum_{t_{1}<t_{k}<t_{2}}\left\|\bar{I}_{k}\left(\bar{x}_{t_{k}}\right)\right\|^{2}+19 \sum_{0<t_{k}<t_{1}}\left\|T_{q}\left(t_{2}-t_{k}\right)-T_{q}\left(t_{1}-t_{k}\right)\right\|^{2}\left\|\bar{I}_{k}\left(\bar{x}_{t_{k}}\right)\right\|^{2}, k=\overline{1, n},
\end{aligned}
$$

since $\left\{T_{q}(t)\right\}_{t \geq 0}$ is strongly continuous and the compactness of $T_{q}(t), t>0$, implies the continuity in the uniform operator topology, it follows that

$$
\mathbb{E}\left\|z\left(t_{2}\right)-z\left(t_{1}\right)\right\|^{2} \rightarrow 0 \text { as } t_{2}-t_{1} \rightarrow 0 .
$$

Hence $\mathrm{V}$ is equicontinuous on $J$.

Finally, we have to show that the map $\Upsilon(\cdot)$ is continuous on $\mathcal{B}_{r}$. Let $\left(x^{n}\right)_{n \in \mathbb{N}}$ be a sequence in $\mathcal{B}_{r}$ and $x \in \mathcal{B}_{r}$ such that $x^{n} \rightarrow x$ in $P C\left(J, \mathcal{L}_{2}\right)$. From the phase space axiom, it is easy to see that $\left(\bar{x}^{n}\right)_{s} \rightarrow \bar{x}_{s}$ as $n \rightarrow \infty$ uniformly for $s \in(-\infty, b]$.

From this fact, hypotheses (H5) and the inequality

$\mathbb{E}\left\|f\left(s, \bar{x}_{\rho\left(s,\left(\bar{x}^{n}\right)_{s}\right)}^{n}\right)-f\left(s, \bar{x}_{\rho\left(s, \bar{x}_{s}\right)}\right)\right\|_{\mathcal{B}}^{2} \leq \mathbb{E}\left\|f\left(s, \bar{x}_{\rho\left(s,\left(\bar{x}^{n}\right)_{s}\right)}^{n}\right)-f\left(s, \bar{x}_{\rho\left(s,\left(\bar{x}^{n}\right)_{s}\right)}\right)\right\|_{\mathcal{B}}^{2}+\mathbb{E}\left\|f\left(s, \bar{x}_{\left.\rho\left(s, \bar{x}^{n}\right)_{s}\right)}\right)-f\left(s, \bar{x}_{\rho\left(s, \bar{x}_{s}\right)}\right)\right\|_{\mathcal{B}}^{2}$, $\mathbb{E}\left\|g\left(s, \bar{x}_{\rho\left(s,\left(\bar{x}^{n}\right)_{s}\right)}^{n}\right)-g\left(s, \bar{x}_{\rho\left(s, \bar{x}_{s}\right)}\right)\right\|_{Q}^{2} \leq \mathbb{E}\left\|g\left(s, \bar{x}_{\rho\left(s,\left(\bar{x}^{n}\right)_{s}\right)}^{n}\right)-g\left(s, \bar{x}_{\left.\rho\left(s, \bar{x}^{n}\right)_{s}\right)}\right)\right\|_{Q}^{2}+\mathbb{E}\left\|g\left(s, \bar{x}_{\rho\left(s,\left(\bar{x}^{n}\right)_{s}\right)}\right)-g\left(s, \bar{x}_{\rho\left(s, \bar{x}_{s}\right)}\right)\right\|_{Q}^{2}$,

$\mathbb{E}\left\|\mathfrak{h}\left(s, \bar{x}_{\left.\rho\left(s, \bar{x}^{n}\right)_{s}\right)}^{n}, \eta\right)-\mathfrak{h}\left(s, \bar{x}_{\rho\left(s, \bar{x}_{s}\right)} \eta\right)\right\|_{Q}^{2} \leq \mathbb{E}\left\|\mathfrak{h}\left(s, \bar{x}_{\rho\left(s,\left(\bar{x}^{n}\right)_{s}\right)}^{n}, \eta\right)-\mathfrak{h}\left(s, \bar{x}_{\left.\rho\left(s, \bar{x}^{n}\right)_{s}\right)}, \eta\right)\right\|_{Q}^{2}+\mathbb{E}\left\|\mathfrak{h}\left(s, \bar{x}_{\rho\left(s, \bar{x}^{n}\right)_{s},}, \eta\right)-\mathfrak{h}\left(s, \bar{x}_{\rho\left(s, \bar{x}_{s}\right)}, \eta\right)\right\|_{Q}^{2}$,

we claim that $f\left(s, \bar{x}_{\rho\left(s,\left(\bar{x}^{n}\right)_{s}\right)}^{n}\right) \rightarrow f\left(s, \bar{x}_{\rho\left(s, \bar{x}_{s}\right)}\right), g\left(s, \bar{x}_{\left.\rho\left(s, \bar{x}^{n}\right)_{s}\right)}^{n}\right) \rightarrow g\left(s, \bar{x}_{\rho\left(s, \bar{x}_{s}\right)}\right)$ and $\mathfrak{h}\left(s, \bar{x}_{\left.\rho\left(s, \bar{x}^{n}\right)_{s}\right)}^{n}\right) \rightarrow \mathfrak{h}\left(s, \bar{x}_{\rho\left(s, \bar{x}_{s}\right)}\right)$ as $n \rightarrow \infty$, for every $s \in J$. Now, the Lebesque dominated convergence theorem (Theorem 2.5) implies that $\Upsilon x^{n} \rightarrow \Upsilon x$ in $\mathcal{B}_{b}^{0}$. Thus $\Upsilon(\cdot)$ is continuous on $\mathcal{B}_{r}$. Then by the Schauder fixed point theorem (Theorem 2.4) $\Upsilon$ has a fixed point and the system (1) has a solution on $J$.

Theorem 3.2 Assume that the hypotheses $(H 0),(H 1)-(H 9)$ and theorem (3.1) are hold, then the system (1) is approximately controllable on $J$.

Proof. Let $\bar{x}^{\epsilon}$ be a fixed point of $\Upsilon$ in $\mathcal{B}_{r}$. Then, we can see that, $\bar{x}^{\epsilon}$ is a mild solution of system (1) under the control

$$
\begin{gathered}
\bar{u}^{\epsilon}(b)=B^{*} T_{q}^{*}(b-t) R\left(\epsilon, \Upsilon_{0}^{b}\right)\left\{x_{b}-C_{q}(b)\left(\phi(0)+\mathrm{m}\left(x_{t_{1}}, \ldots, x_{t_{m}}\right)(0)\right)-S_{q}(b) \xi\right. \\
-\int_{0}^{b} T_{q}(b-\tau) f\left(\tau, \bar{x}_{\rho\left(\tau, \bar{x}_{\tau}\right)}^{\epsilon}\right) d s-\int_{0}^{b} T_{q}(b-\tau)\left[\int_{-\infty}^{\tau} g\left(\tau, \bar{x}_{\rho\left(\tau, \bar{x}_{\tau}\right)}^{\epsilon}\right) d \omega(\tau)\right] d \tau \\
\left.\left.-\int_{0}^{b} \int_{Z} T_{q}(b-\tau) \mathfrak{h}\left(\tau, \bar{x}_{\rho\left(\tau, \bar{x}_{\tau}\right)}^{\epsilon}, \eta\right) \widetilde{N}(d \tau, d \eta)-\sum_{0<t_{k}<b} T_{q}\left(b-t_{k}\right) I_{k}\left(\bar{x}_{t_{k}}^{\epsilon}\right)-\sum_{0<t_{k}<b} T_{q}\left(b-t_{k}\right) \bar{I}_{k}\left(\bar{x}_{t_{k}}^{\epsilon}\right)\right)\right\}, k=\overline{1, n},
\end{gathered}
$$


and satisfies

$$
\begin{aligned}
& \bar{x}^{\epsilon}(b)=C_{q}(b)\left(\phi(0)+\mathrm{m}\left(x_{t_{1}}, \ldots, x_{t_{m}}\right)(0)\right)+S_{q}(b) \xi+\int_{0}^{b} T_{q}(b-s) f\left(s, \bar{x}_{\rho\left(s, \bar{x}_{s}\right)}^{\epsilon}\right) d s \\
& +\int_{0}^{b} T_{q}(b-s) B \bar{u}^{\epsilon}(s) d s+\int_{0}^{b} T_{q}(b-s)\left[\int_{-\infty}^{s} g\left(s, \bar{x}_{\rho\left(s, \bar{x}_{s}^{\epsilon}\right)}^{\epsilon}\right) d \omega(s)\right] d s \\
& +\int_{0}^{b} \int_{Z} T_{q}(b-s) \mathfrak{h}\left(s, \bar{x}_{\rho\left(s, \bar{x}_{s}^{\epsilon}\right)}^{\epsilon}, \eta\right) \widetilde{N}(d s, d \eta)+\sum_{0<t_{k}<b} T_{q}\left(b-t_{k}\right) I_{k}\left(\bar{x}_{t_{k}}^{\epsilon}\right) \\
& +\sum_{0<t_{k}<b} T_{q}\left(b-t_{k}\right) \bar{I}_{k}\left(\bar{x}_{t_{k}}^{\epsilon}\right), k=\overline{1, n} . \\
& \bar{x}^{\epsilon}(b)=C_{q}(b)\left(\phi(0)+\mathrm{m}\left(x_{t_{1}}, \ldots, x_{t_{m}}\right)(0)\right)+S_{q}(b) \xi \\
& +\int_{0}^{b} T_{q}(b-s) f\left(s, x_{\rho\left(s, \bar{x}_{s}^{\epsilon}\right)}\right) d s+\int_{0}^{b} T_{q}(b-s) B\left[B^{*} T_{q}^{*}(b-t) R\left(\epsilon, \Upsilon_{0}^{b}\right)\right. \\
& \times\left\{x_{b}-C_{q}(b)\left(\phi(0)+\mathrm{m}\left(x_{t_{1}}, \ldots, x_{t_{m}}\right)(0)\right)-S_{q}(b) \xi\right. \\
& -\int_{0}^{b} T_{q}(b-\tau) f\left(\tau, \bar{x}_{\rho\left(\tau, \bar{x}_{\tau}\right)}^{\epsilon}\right) d s-\int_{0}^{b} T_{q}(b-\tau)\left[\int_{-\infty}^{\tau} g\left(\tau, \bar{x}_{\rho\left(\tau, \bar{x}_{\tau}^{\epsilon}\right)}^{\epsilon}\right) d \omega(\tau)\right] d \tau \\
& -\int_{0}^{b} \int_{Z} T_{q}(b-\tau) \mathfrak{h}\left(\tau, \bar{x}_{\rho\left(\tau, \bar{x}_{\tau}\right)}^{\epsilon}, \eta\right) \widetilde{N}(d \tau, \mathrm{d} \eta)-\sum_{0<t_{k}<b} T_{q}\left(b-t_{k}\right) I_{k}\left(\bar{x}_{t_{k}}^{\epsilon}\right) \\
& \left.\left.\left.-\sum_{0<t_{k}<b} T_{q}\left(b-t_{k}\right) \bar{I}_{k}\left(\bar{x}_{t_{k}}^{\epsilon}\right)\right)\right\}\right] d s+\int_{0}^{b} T_{q}(b-s)\left[\int_{-\infty}^{s} g\left(s, \bar{x}_{\rho\left(s, \bar{x}_{s}^{\epsilon}\right)}^{\epsilon}\right) d \omega(s)\right] d s \\
& +\int_{0}^{b} \int_{Z} T_{q}(b-s) \mathfrak{h}\left(s, \bar{x}_{\rho\left(s, \bar{x}_{s}\right)}^{\epsilon}, \eta\right) \widetilde{N}(d s, d \eta)+\sum_{0<t_{k}<b} T_{q}\left(b-t_{k}\right) I_{k}\left(\bar{x}_{t_{k}}^{\epsilon}\right) \\
& +\sum_{0<t_{k}<b} T_{q}\left(b-t_{k}\right) \bar{I}_{k}\left(\bar{x}_{t_{k}}^{\epsilon}\right), k=\overline{1, n}, \\
& =x_{b}+\left(\Upsilon_{0}^{b} R\left(\epsilon, \Upsilon_{0}^{b}\right)-I\right)\left\{x_{b}-C_{q}(b)\left(\phi(0)+\mathrm{m}\left(x_{t_{1}}, \ldots, x_{t_{m}}\right)(0)\right)-S_{q}(b) \xi\right. \\
& -\int_{0}^{b} T_{q}(b-\tau) \mathrm{f}\left(\tau, \bar{x}_{\rho\left(\tau, \bar{x}_{\tau}\right)}^{\epsilon}\right) d s-\int_{0}^{b} T_{q}(b-\tau)\left[\int_{-\infty}^{\tau} g\left(\tau, \bar{x}_{\rho\left(\tau, \bar{x}_{\tau}^{\epsilon}\right)}^{\epsilon}\right) d \omega(\tau)\right] d \tau \\
& -\int_{0}^{b} \int_{Z} T_{q}(b-\tau) \mathfrak{h}\left(\tau, \bar{x}_{\rho\left(\tau, \bar{x}_{\tau}\right)}^{\epsilon}, \eta\right) \widetilde{N}(d \tau, d \eta)-\sum_{0<t_{k}<b} T_{q}\left(b-t_{k}\right) I_{k}\left(\bar{x}_{t_{k}}^{\epsilon}\right) \\
& \left.\left.-\sum_{0<t_{k}<b} T_{q}\left(b-t_{k}\right) \bar{I}_{k}\left(\bar{x}_{t_{k}}^{\epsilon}\right)\right)\right\}, k=\overline{1, n} . \\
& =x_{b}-\epsilon R\left(\epsilon, \Upsilon_{0}^{b}\right)\left\{x_{b}-C_{q}(b)\left(\phi(0)+\mathrm{m}\left(x_{t_{1}}, \ldots, x_{t_{m}}\right)(0)\right)-S_{q}(b) \xi\right. \\
& -\int_{0}^{b} T_{q}(b-\tau) f\left(\tau, \bar{x}_{\rho\left(\tau, \bar{x}_{\tau}^{\epsilon}\right)}^{\epsilon}\right) d s-\int_{0}^{b} T_{q}(b-\tau)\left[\int_{-\infty}^{\tau} g\left(\tau, \bar{x}_{\rho\left(\tau, \overline{x_{\tau}}\right.}^{\epsilon}\right) d \omega(\tau)\right] d \tau
\end{aligned}
$$

$\left.\left.-\int_{0}^{b} \int_{Z} T_{q}(b-\tau) \mathfrak{h}\left(\tau, \bar{x}_{\rho\left(\tau, \bar{x}_{\tau}\right)}^{\epsilon}, \eta\right) \widetilde{N}(d \tau, d \eta)-\sum_{0<t_{k}<b} T_{q}\left(b-t_{k}\right) I_{k}\left(\bar{x}_{t_{k}}^{\epsilon}\right)-\sum_{0<t_{k}<b} T_{q}\left(b-t_{k}\right) \bar{I}_{k}\left(\bar{x}_{t_{k}}^{\epsilon}\right)\right)\right\}, k=\overline{1, n}$.

The uniform boundedness of the functions $f, g$ and $\mathfrak{h}$ imply that the sequences $\left\{f\left(s, \bar{x}_{\rho\left(s, \bar{x}_{s}^{\epsilon}\right)}^{\epsilon}\right)\right\},\left\{g\left(s, \bar{x}_{\rho\left(s, \bar{x}_{s}^{\epsilon}\right)}^{\epsilon}\right)\right\}$ and $\left\{\mathfrak{h}\left(s, \bar{x}_{\rho\left(s, \bar{x}_{s}^{\epsilon}\right)}^{\epsilon}\right), \eta\right\}$ are bounded in $\mathcal{L}_{2}(J, \mathcal{B})$. Hence the subsequence, denoted by $\left\{f\left(s, \bar{x}_{\rho\left(s, \bar{x}_{s}\right)}^{\epsilon}\right)\right\},\left\{g\left(s, \bar{x}_{\rho\left(s, \bar{x}_{s}^{\epsilon}\right)}^{\epsilon}\right)\right\}$ and $\left\{\mathfrak{h}\left(s, \bar{x}_{\rho\left(s, \bar{x}_{s}\right)}^{\epsilon}\right), \eta\right\}$ that weakly converges to, say $f(s), g(s)$ and $\mathfrak{h}(s)$ in $\mathcal{L}_{2}(J, H)$ and $\mathcal{L}_{2}\left(J, \mathcal{L}_{Q}(K, H)\right)$. Then it is easy to see that

$$
\mathbb{E}\left\|\int_{0}^{b} T_{q}(b-s)\left[f\left(s, \bar{x}_{\rho\left(s, \bar{x}_{s}^{\epsilon}\right)}^{\epsilon}\right)-f(s)\right] d s\right\|^{2} \leq \int_{0}^{b}\left\|T_{q}(b-s)\right\|^{2} \mathbb{E}\left\|f\left(s, \bar{x}_{\rho\left(s, \bar{x}_{s}^{\epsilon}\right)}^{\epsilon}\right)-f(s)\right\|^{2} d s \rightarrow 0,
$$

$\mathbb{E}\left\|\int_{0}^{b} T_{q}(b-s)\left[\int_{-\infty}^{s}\left[g\left(s, \bar{x}_{\rho\left(s, \bar{x}_{s}^{\epsilon}\right)}^{\epsilon}\right)-g(s)\right]\right] d \omega(s)\right\|_{Q}^{2} \leq \int_{0}^{b}\left\|T_{q}(b-s)\right\|^{2}\left[\int_{-\infty}^{s} \mathbb{E}\left\|g\left(s, \bar{x}_{\rho\left(s, \bar{x}_{s}^{\epsilon}\right)}^{\epsilon}\right)-g(s)\right\|_{Q}^{2} d \omega(s) \rightarrow 0\right.$,

$$
\begin{gathered}
\mathbb{E}\left\|\int_{0}^{b} T_{q}(t-s)\left[\int_{Z}\left[\mathfrak{h}\left(s, \bar{x}_{\rho\left(s, \bar{x}_{s}^{\epsilon}\right)}^{\epsilon}, \eta\right)-\mathfrak{h}(s)\right]\right] \widetilde{N}(d s, d \eta)\right\|_{Q}^{2} \\
\leq \int_{0}^{b}\left\|T_{q}(b-s)\right\|^{2}\left[\int_{Z} \mathbb{E}\left\|\mathfrak{h}\left(s, \bar{x}_{\rho\left(s, \bar{x}_{s}^{\epsilon}\right.}^{\epsilon}, \eta\right)-\mathfrak{h}(s)\right\|_{Q}^{2}\right] \widetilde{N}(d s, d \eta) \rightarrow 0
\end{gathered}
$$

As $\epsilon \rightarrow 0^{+}$and the boundedness of $I$ and $\bar{I}$ implies that

$$
E\left\|\bar{x}^{\epsilon}(b)-x_{b}\right\|^{2} \leq \mathbb{E} \| \epsilon R\left(\epsilon, \Upsilon_{0}^{b}\right)\left\{x_{b}-C_{q}(t)\left(\phi(b)+\mathrm{m}\left(x_{t_{1}}, \ldots, x_{t_{m}}\right)(0)\right)-S_{q}(b) \xi\right.
$$




$$
\begin{gathered}
-\int_{0}^{b} T_{q}(b-s) f\left(s, \bar{x}_{\rho\left(s, \bar{x}_{s}^{\epsilon}\right)}^{\epsilon}\right) d s-\int_{0}^{b} T_{q}(b-s)\left[\int_{-\infty}^{s} g\left(s, \bar{x}_{\rho\left(s, \bar{x}_{s}^{\epsilon}\right)}^{\epsilon}\right) d \omega(s)\right] d s \\
-\int_{0}^{b} \int_{Z} T_{q}(b-s) \mathfrak{h}\left(s, \bar{x}_{\rho\left(s, \bar{x}_{s}^{\epsilon}\right)}^{\epsilon}, \eta\right) \widetilde{N}(d s, d \eta)-\sum_{0<t_{k}<b} T_{q}\left(b-t_{k}\right) I_{k}\left(\bar{x}_{t_{k}}^{\epsilon}\right) \\
\left.\left.-\sum_{0<t_{k}<b} T_{q}\left(b-t_{k}\right) \bar{I}_{k}\left(\bar{x}_{t_{k}}^{\epsilon}\right)\right)\right\} \|^{2} \rightarrow 0 \text { as } \epsilon \rightarrow 0^{+} .
\end{gathered}
$$

Hence $\bar{x}^{\epsilon}(b) \rightarrow x_{b}$ holds in $H$ and consequently we get the approximate controllability of system (1). 圆

\section{Application}

In this section, we can apply the previous results to study the approximate controllability of fractional impulsive stochastic differential system of order $1<q \leq 2$ with nonlocal, state-dependent delay and Poisson jumps of the form

$$
\begin{gathered}
{ }^{c} D_{t}^{q} z(t, x)=\frac{\partial^{2}}{\partial x^{2}} z(t, x)+\mu(t, x)+\left(\int_{-\infty}^{t} a_{1}(s-t) z\left(s-\rho_{1}(t) \rho_{2}(\|z(t)\|), x\right) d s\right)+\left(\int_{-\infty}^{t} a_{2}(s-t) z\left(s-\rho_{1}(t) \rho_{2}(\|z(t)\|), x\right) d s\right) \partial \beta(t), \\
+\int_{Z} \eta\left(\int_{-\infty}^{t} a_{3}(s-t) z\left(s-\rho_{1}(t) \rho_{2}(\|z(t)\|), x\right) d s\right) \widetilde{N}(d t, d \eta), \\
t \in J=[0, b] \backslash\left\{t_{1}, t_{2}, \ldots, t_{m}\right\},(t, x) \in J \times[0, \pi], \\
z(t, 0)=z(t, \pi)=0, t \in J \\
z(t, x)=\phi(t, x)+\sum_{i=0}^{m} C_{i} z\left(t_{i}+x\right), t \leq 0, x \in[0, \pi], t \in(-\infty, 0], \\
I_{k}\left(z\left(t_{k}\right)\right) x=\int_{-\infty}^{t_{k}} c_{k}\left(t_{k}-s\right) z(s, x) d s, x \in[0, \pi], k=\overline{1, n}, \\
\bar{I}_{k}\left(z\left(t_{k}\right)\right) x=\int_{-\infty}^{t_{k}} d_{k}\left(t_{k}-s\right) z(s, x) d s, x \in[0, \pi], k=\overline{1, n},
\end{gathered}
$$

where ${ }^{c} D_{t}^{q}$ is the Caputo fractional partial derivative of order $1<q \leq 2, \mu:[0,1] \times(0, \pi) \rightarrow(0, \pi)$ is continuous, and $c_{k}, d_{k}>0$ for $k=1,2, \ldots, n$. Also $0<t_{1}<t_{2}<\cdots<$ $t_{m}<b$ are prefixed numbers. $\rho_{i}:[0, \infty) \rightarrow[0, \infty), i=$ 1,2. $a_{1}, a_{2}, a_{3}: \mathbb{R} \rightarrow \mathbb{R}$ are continuous. $\phi \in \mathcal{B}=P C_{0} \times$ $\mathcal{L}_{2}(\mathrm{~g}, H)$ (gis a positive function on $(-\infty,-r)$ ) be the phase space in the Hale et al [20]. The operator $A$ is defined by $A \xi=\frac{\partial^{2}}{\partial x^{2}} \xi$ with domain $D(A)=\left\{\xi \in H, \frac{d \xi}{d x}\right.$, are absolutely continuous and $\left.\left(\frac{d^{2}}{d x^{2}}\right) \xi \in H \quad \xi(0)=\xi(\pi)=0\right\}$. Also $\beta(t)$ enotes a standard one-dimensional Wiener process defined on a stochastic basis $\left(\Omega, \mathfrak{F}_{,}\left\{\mathfrak{F}_{t}\right\}_{t \geq 0}, \mathbb{P}\right)$. To write the above system (3) into the abstract form (1), we can choose the space. $H=U=\mathcal{L}_{2}([0, \pi])$. Define $A: H \rightarrow H \quad$ by $A x=x^{\prime \prime}(A z)(x)=\frac{\partial^{2} z(x)}{\partial x^{2}}$ with the domain $D(A)=\{x \in$ $H: x, x^{\prime}$ are absolutely continuous, $x^{\prime \prime} \in H$ and $x(0)=$ $x(\pi)=0\}$ and for $z \in D(A)$. Also the set $\left\{e_{n}, n \in \mathbb{N}\right\}$ is an orthonormal basis of $H$. In particular $A x=\sum_{n=1}^{\infty}-$ $n^{2}\left\langle x, e_{n}\right\rangle e_{n}, x \in D(A)$. Then $A$ is the infinitesimal generator of a strongly continuous cosine family $C(t) x=\sum_{n=1}^{\infty} \cos (n t)\left\langle x, e_{n}\right\rangle e_{n,}, t \in \mathbb{R}$ and the associated sine family in $H$ defined by $S(t) x=\sum_{n=1}^{\infty} \frac{\sin (n t)}{n}\left\langle x, e_{n}\right\rangle e_{n}, t \in \mathbb{R}$. From [37], for all $x \in H, t \in \mathbb{R},\left\|S_{q}(t)\right\| \leq 1$ and $\left\|C_{q}(t)\right\| \leq 1$.

Hence the spectrum $A$ consists of eigen values $n$ for $n \in \mathbb{N}$, with associated eigen vectors $e_{n}(x)=\sqrt{\frac{2}{\pi}} \sin (n x), n \in$ $\mathbb{N}$ Define an infinite dimensional space $U$ by $U=\{u=$ $\left.\sum_{n=2}^{\infty} u_{n} e_{n}(x) \mid \sum_{n=2}^{\infty} u_{n}^{2}<\infty\right\}, 0 \leq x \leq \pi$, and the norm in $U$ is $\|u\|=\left(\sum_{n=2}^{\infty} u_{n}^{2}\right)^{\frac{1}{2}}$. The bounded linear operator
$B: U \rightarrow H$ is defined by $B u(t)(x)=\mu(t, x), 0 \leq x \leq \pi$. The functions $f, g$ and $\mathfrak{h}$ are continuous. Let $(\Omega, \mathfrak{F}, \mathbb{P})$ be a complete probability space and $\{K(t): t \in J\}$ is a Poisson point process taking values in the space $K=[0, \infty)$ with a $\sigma$-finite intensity measure $\lambda(d y)$, The Poisson counting measure $\widetilde{N}(d t, d y)$ is induced by $\mathrm{K}(\cdot)$ and the compensating martingale measure is denoted by

$$
\widetilde{N}(d t, d y):=N(d t, d y)-d t \lambda(d y) .
$$

For $q \in(1,2), A$ generates a strongly continuous cosine family $C(t)$, it follows from the subordinate principle (see Theorem 3.1, [6]) that $A$ generates a strongly continuous exponentially bounded fractional cosine family $C_{q}(t)$ such that $C_{q}(0)=I$ and

$$
\begin{gathered}
C_{q}(t)=\int_{0}^{\infty} \varphi_{t, \frac{q}{2}}(s) C(s) d s, t>0, \\
\text { where } \varphi_{t, \frac{q}{2}}(s)=t^{-\frac{q}{2}} \varrho_{\frac{q}{2}}\left(s t^{-\frac{q}{2}}\right) \text { and } \\
\varrho_{\vartheta}(x)=\sum_{n=0}^{\infty} \frac{(-x)^{n}}{n ! \Gamma(-\vartheta n+1-\theta)},(0<\theta<1) .
\end{gathered}
$$

It is clear that the fractional differential system (2) is approximately controllable on $J$ for $q=2$. Hence for $q=2$ with the above choices, the system (3) can be rewritten to the abstract form (1) and all the conditions of Theorem 3.1 are satisfied. Thus there exists mild solutions for the system (3). Moreover all the conditions of Theorem 3.2 are satisfied and hence the fractional stochastic differential equations with Poisson jumps (3) is approximately controllable on $J$.

We have to use the following results:

(I) the functions $a_{1}: \mathbb{R} \rightarrow \mathbb{R}$ is continuous, bounded and 
$\mathcal{L}_{f}=\left(\int_{-\infty}^{0} \frac{a_{1}^{2}(s)}{\mathrm{g}(s)} d s\right)^{\frac{1}{2}}<\infty$.

(ii) the functions $a_{2}: \mathbb{R} \rightarrow \mathbb{R}$ is continuous, bounded and $\mathcal{L}_{g}=\left(\int_{-\infty}^{0} \frac{a_{2}^{2}(s)}{\mathrm{g}(s)} d s\right)^{\frac{1}{2}}<\infty$.

(iii) the functions $a_{3}: \mathbb{R} \rightarrow \mathbb{R}$ is continuous, bounded and $\mathcal{L}_{\mathfrak{h}}=\left(\int_{-\infty}^{0} ? \frac{a_{3}^{2}(s)}{\mathrm{g}(s)} d s\right)^{\frac{1}{2}}<\infty$.

(iv) the function $c_{k}: \mathbb{R} \rightarrow \mathbb{R}$ is continuous such that, $\mathcal{L}_{c_{k}}=\left(\int_{-\infty}^{0} \frac{\left(c_{k}(-s)\right)^{2}}{\mathrm{~g}(s)} d s\right)^{\frac{1}{2}}<\infty$ for every $k=1,2 \ldots n$.

(v) the function $d_{k}: \mathbb{R} \rightarrow \mathbb{R}$ is continuous such that, $\mathcal{L}_{d_{k}}=\left(\int_{-\infty}^{0} \frac{\left(d_{k}(-s)\right)^{2}}{\mathrm{~g}(s)} d s\right)^{\frac{1}{2}}<\infty$ for every $k=1,2 \ldots n$.

By defining the functions $f, \mathfrak{h}, \rho, I_{k}, \bar{I}_{k}: \mathcal{B} \rightarrow H$ and $g: \mathcal{B} \rightarrow \mathcal{L}_{Q}(H)$ by

$$
\begin{gathered}
\rho(t, \psi)=\rho_{1}(t) \rho_{2}(\|\psi(0)\|), \\
f(\psi)(x)=\int_{-\infty}^{0} a_{1}(s) \psi(s, x) d s, \\
g(\psi)(x)=\int_{-\infty}^{0} a_{2}(s) \psi(s, x) d \omega(s), \\
\mathfrak{h}(\psi)(x)=\int_{-\infty}^{t} a_{1}(s) \psi(s, x) d s, \\
I_{k}(\psi)(x)=\int_{-\infty}^{t} c_{k}(-s) \psi(s, x) d s, k=1,2, \ldots, n, \\
\bar{I}_{k}(\psi)(x)=\int_{-\infty}^{t} d_{k}(-s) \psi(s, x) d s, k=1,2, \ldots, n .
\end{gathered}
$$

Moreover, the maps $f, g, \mathfrak{h}, I_{k}$ and $\bar{I}_{k}$ are bounded linear operators $\|f\|^{2} \leq \mathcal{L}_{f},\|g\|_{Q}^{2} \leq \mathcal{L}_{g},\|\mathfrak{h}\|_{Q}^{2} \leq \mathcal{L}_{\mathfrak{h}}, \quad\left\|I_{k}\right\|^{2} \leq$ $\mathcal{L}_{c_{k}}$ and $\left\|\bar{I}_{k}\right\|^{2} \leq \mathcal{L}_{d_{k}}$.

Because of the compactness of $T_{q}(t)$ generated by $A$, the associated linear system (2) is approximately controllable [30]. Hence by Theorem (3.2), the system (3) is approximately controllable.

\section{Conclusion}

This paper has investigated the existence of mild solutions and approximate controllability of second order $(q \in(1,2))$ fractional impulsive stochastic differential system with nonlocal, state-dependent delay and Poisson umps in Hilbert spaces. For the future work, the controllability and stability results could be extended to study the neutral impulsive fractional stochastic differential systems with state-dependent delay and Poisson jumps satisfying the nonlocal condition according to the method in this paper. Hence our main results are the generalization of the recent results on fractional stochastic control systems with state-dependent delay and Poisson jumps.

\section{References}

[1] K. J. Astrom, Introduction to stochastic control theory, AcademicPress, NewYork, 1970.

[2] H. M. Ahmeda, M. M. El-Borai, H. M. El-Owaidy and A. S. Ghanema, Null controllability of fractional stochastic delay integrodifferential equations, J. Math. Computer Sci., 19 (2019), 143-150.
[3] K. Aissani and M. Benchohra, Fractional Integro-Differential Equations with State-Dependent Delay Advances in Dynamical Systems and Applications 9, 1 (2014), 17-30.

[4] B. D. Andrade and J. P. C. D. Santos, Existence of solutions for a fractional neutral integro-differential equations with unbounded delay, Electron. J. Differ. Equ., 90 (2012), 1-13.

[5] R. P. Agarwal, J. P. C. dos Santos and C. Cuevas, Analytic resolvent operator and existence results for fractional integro-differetial equations, J. Abstr. Differ. Equ. Appl., 2, 2 (2012), 26-47.

[6] E. G. Bazhlekova, Fractional evolution equtions in Banach spaces, Diss. University Press Facilities, Eindhoven University of Technology, 2001.

[7] M. Benchohra and F. Berhoun, Impulsive fractional differential equations with state-dependent delay, Communications in Appl. Anal., 14, 2 (2010), 213-224.

[8] O. Bolojan-Nica, G. Infante and R. Precup, Existence results for systems with coupled nonlocal initial conditions, Nonlinear Anal., 94 (2014), 231-242.

[9] A. E. Bashirov and N. I. Mahmudov, On concepts of controllability for linear deterministic and stochastic systems, SIAM J. Control Optim., 37 (1999), 1808--1821.

[10] A. Boudaoui and A. Slama, Approximate Controllability of Nonlinear Fractional Impulsive Stochastic Differential Equations with Nonlocal Conditions and Infinite Delay, Nonlinear Dyn. Syst. Theory, 1 (2016) 35-48.

[11] P. Balasubramaniam, V. Vembarasan and T. Senthilkumar, Approximate controallability of impulsive fractional integro-differential systems with nonlocal conditions in Hilbert space, Numer. Funct. Anal. Optimi., 35 (2014), 177-197.

[12] A. Chadha, S. N. Bora and R. Sakthivel, Approximate controllability of impulsive stochastic fractional differential equations with nonlocal conditions, Dyn. Syst. Appl., 1 (2018), 1-29.

[13] R. Chaudhary and D. N. Pandey, Existence results for a class of impulsive neutral fractional stochastic integro-differential systems with state dependent delay, Stochastic Anal. Appl., 2019, Taylor and Francis Group.

[14] J. Chu and P. J. Torres, Applications of schauder's fixed point theorem to singular differential equations, Bull. London Math. Soc., 39 (2007), 653-660.

[15] S. Deng, X. Shu and J. Mao Existence and exponential stability for impulsive neutral stochastic functional differential equations driven by $\mathrm{fBm}$ with noncompact semigroup via Mönch fixed point J. Math. Anal. Appl., !67 (2018), 398-420.

[16] G. Da Prato and J. Zabczyk, Stochastic Equations in Infinite Dimensions, Cambridge University Press, Cambridge, 1992.

[17] H. O. Fattorimi, Second order linear differential equations in Banach spaces, North Holland, Amsterdam, New York, and London (1985).

[18] B. Goldys and B. Malsowski, Exponential ergodicity for stochastic Burgers and 2D Navier-Stokes equations, J. Funct. Anal., 226 (2005), 230-255.

[19] T. Guendouzi and O. Benzatout, Existence of Mild Solutions for Impulsive Fractional Stochastic Differential Inclusions with State-Dependent Delay, Hindawi Publishing Corporation, Chinese Journal of Mathematics, Volume 2014, Article ID 981714, 13 pages. 
[20] J. K. Hale and J. Kato, Phase space for retarded equations with infinite delay, Fumkcial Ekvac., 21 (1978), 11-41.

[21] E. Hernandez and M. McKibben, On state-dependent delay partial neutral functional differential equations, Appl. Math. Comput., 186 (2007), 294--301.

[22] E. Hernandez, M. McKibben and H. Henriquez, Existence results for partial neutral functional differential equations with state-dependent delay, Math. Comput. Model., 49 (2009), $1260-1267$.

[23] E. Hernandez, M. Pierri and G. Goncalves, Existence Results for an Impulsive Abstract Partial Differential Equation with State-Dependent Delay, Com. Math. Appl., 52 (2006), 411420.

[24] E. Hernandez and H. R. Henriquez, Existence results for partial neutral functional differential equations with unbounded delay J. Math. Anal. Appl., 221 (1998), 452-475.

[25] R. S. Jain and M. B. Dhakne, On global existence of solutions for abstract nonlinearfunctional integro-differential equations with nonlocal condition, Contempoary Math. and stat., 1 (2013), 44-53.

[26] P. Kalamani, D. Baleanu, S. Selvarasu and M. Mallika Arjunan, On existence results for impulsive fractional neutral stochastic integro-differential equations with nonlocal and state-dependent delay conditions,. Adv. Differ. Equ.,(2016) 2016: 163.

[27] L. Kexue, P. Jigen and G. Jinghuai, Controllability of nonlocal fractional differential systems of order $\alpha \in(1,2]$ in Banach spaces, Rep. Math. Physics, 71, 1 (2013), 33-43.

[28] J. A. Kilbas, H. Srivastava and J. J. Trujillo, Theory and Applications of Fractional DifferentialEquations, Elsevier, Amesterdam, 2006.

[29] W. Mao, L. Hu and X. Mao, Neutral stochastic functional differential equations with Levy jumps under the local Lipschitz condition, Advances in Difference Equations (2017) 2017: 57.

[30] P. Muthukumar and C. Rajivganthi, Approximate controllability of impulsive neutral stochastic functional differential system with state-dependent delay in Hilbert spaces, J. Control Theory Appl., 11, 3 (2013), 351-358.

[31] K. S. Miller and B. Ross, An introduction to the fractional calculus and fractional differential equations, John Wiley and Sons Inc., NewYork, 1993.

[32] P. Muthukumar and K. Thiagu, Existence of solutions and approximate controllability of fractional nonlocal stochastic differential equations of order $1<q \leq 2$ with infinite delay and Poisson jumps, Differ. Eqn. Syst.

[33] P. Muthukumar and K. Thiagu, Existence of solutions and approximate controllability of fractional nonlocal neutral impulsive stochastic differential equations of order $1<q<2$ with infinite delay and Poisson jumps, J. dyn. control syst., (2015): $1-23$.

[34] B. Oksendal, Stochastic differential equations: An introduction with applications, Springer-verlag, 1995.

[35] I. Podlubny, Fractional differential equations: an introduction to fractional derivatives, fractional differential equations, to methods of their solution and some of their applications, Vol. 198, Academic Press, New York, 1999.
[36] A. Pazy, Semigroups of linear operators and applications to partial differential equations, Springer-Verlag, New York, 1983.

[37] Y. Ren and R. Sakthivel, Existence, uniqueness, and stability of mild solutions for Second-order neutral stochastic evolution equations with infinite delay and Poisson jumps, J. Math. Phys. 53, 7 (2012), 073517.

[38] C. Rajivganthi, K. Thiagu, P. Muthukumar and P. Balasubramaniam, Existence of solutions and approximate controllability of impulsive fractional stochastic differential systems with infinite delay and poisson jumps, Appl. Math., 4 (2015), 395-419.

[39] R. Sakthivel and E. R. Anandhi, Approximate controllability of impulsive differential equations with state-dependent delay, Int. J. Con., 83 (2010), 387-393.

[40] A. Slama and A. Boudaoui, Existence of solutions for nonlinear fractional impulsive stochastic differential equations with nonlocal conditions and infinite delay, Int. J. differ. eqn. appl., 4 (2014), 185-201.

[41] S. G. Samko, A. A. Kilbas and O. I. Marichev, Fractional Integrals and Derivatives: Theory and Applications, Gordon and Breach Science Publisher, Yverdon, 1993.

[42] S. Selvarasu, P. Kalamani and M. Mallika Arjunan, Approximate controllability of impulsive fractional stochastic integro-differential systems with state-dependent delay and poisson jumps, J. Fractional Calculus and Applications, 2 (2018), 229-254.

[43] R. Sakthivel and Y. Ren, Approximate controllability of fractional differential equations with state-dependent delay, Results Math., 63 (2013), 949-963.

[44] K. Thiagu and P. Muthukumar, Non-Lipschitz Sobolev Type Fractional Neutral Impulsive Stochastic Differential Equations with Fractional Stochastic Nonlocal Condition, Infinite Delay and Poisson Jumps, Compu. Appl. Math. Journal, 6, 1 (2020), 1-11.

[45] N. Valliammal, C. Ravichandran, Z. Hammouch and H. M. Baskonu A New Investigation on Fractional-Ordered Neutral Differential Systems with State-Dependent Delay, Int. J. of Nonlinear Sci. Numer. simul., 20, 7-8 (2019).

[46] J. Wang and H. M. Ahmed, Null controllability of nonlocal Hilfer fractional stochastic differential equations, Miskolc Math. Notes, 18 (2017), 1073-1083.

[47] T. Yang, Impulsive systems and control: Theory and applications, Springer-Verlag, Berlin, 2001.

[48] Z. Yan and H. Zhang, Asymptotic stability of fractional impulsive neutral stochastic partial integro-differential equations with state-dependent delay, Elect. J. Diff. Equ., 206 (2013) 1-20.

[49] Y. Zang and J. Li, Approximate controllability of fractional impulsive neutral stochastic differential equations with nonlocal conditions, Bound. Value Probl., 2013, 193.

[50] X. Zhang, C. Zhu and C. Yuan, Approximate controllability of fractional impulsive evolution systems involving nonlocal initial conditions, Adv. Diff. Equ., 2015, pp 14.

[51] X. Zhang, C. Zhu and C. Yuan, Approximate controllability of impulsive fractional stochastic differential equations with state-dependent delay, Adv. Diff. Equ., 2015, pp-12. 